\title{
Dysprosium-based experimental representatives of an Ising-Heisenberg chain and a decorated Ising ring
}

\author{
Willem Van den Heuve ${ }^{*}$ and Liviu F. Chibotaru ${ }^{\dagger}$ \\ Chemistry Department, Katholieke Universiteit Leuven, Celestijnenlaan 200F, B-3001 Leuven, Belgium
}

(Dated: July 7, 2021)

\begin{abstract}
It is shown that the bond-decorated Ising model is a realistic model for certain real magnetic compounds containing lanthanide ions. The lanthanide ion plays the role of Ising spin. The required conditions on the crystal-field spectrum of the lanthanide ion for the model to be valid are discussed and found to be in agreement with several recent $a b$ initio calculations on $\mathrm{Dy}^{3+}$ centers. Similarities and differences between the spectra of the simple Ising chain and the decorated Ising chain are discussed and illustrated, with attention to level crossings in a magnetic field. The magnetic properties of two actual examples (a $[\mathrm{DyCuMoCu}]_{\infty}$ chain and $\mathrm{a} \mathrm{Dy}_{4} \mathrm{Cr}_{4}$ ring) are obtained by a transfer-matrix solution of the decorated Ising model. $g$-factors of the metal ions are directly imported from $a b$ initio results, while exchange coupling constants are fitted to experiment. Agreement with experiment is found to be satisfactory, provided one includes a correction (from ab initio results) for susceptibility and magnetization to account for the presence of excited Kramers doublets on $\mathrm{Dy}^{3+}$.

PACS numbers: $75.50 . \mathrm{Xx}, 75.10 . \mathrm{Pq}$
\end{abstract}

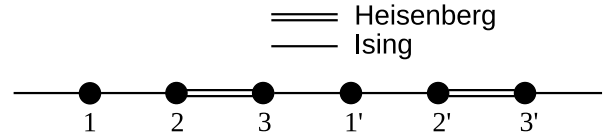

FIG. 1. The Ising-Heisenberg chain discussed in Ref. 3 (showing only two unit cells). The bonds of the Ising chain $\left(1-1^{\prime}-\right.$ $\left.1^{\prime \prime}-\ldots\right)$ are decorated with Heisenberg dimers $\left((2-3),\left(2^{\prime}-3^{\prime}\right)\right.$, ...). The partition function of this chain is exactly solvable.

\section{INTRODUCTION}

In a 1959 paper M. E. Fisher introduced the general bond-decorated Ising model as one example of a set of exactly solvable transformations of spin-1/2 Ising models. 1 A bond-decorated Ising model has an "arbitrary statistical mechanical system" inserted in every original Ising bond. The partition function of this decorated model is related to the partition function of the original or bare Ising model by the addition of a prefactor and a renormalization of the coupling constants and magnetic moments (the Ising model is supposed to be in a parallel magnetic field) $! 2$ Knowledge of the partition function of a given Ising model thus allows one to obtain the partition function of any bond-decorated version of that Ising model.

More recently, Strečka and Jaščur have used the method of bond-decoration to investigate the thermodynamics of mixed Ising-Heisenberg chains in parallel magnetic fields where the decorating unit is a spin dimer or trimer with anisotropic Heisenberg coupling (see Fig. 1) ${ }^{3 / 4}$ The partition function of these chains is readily obtained from the known partition function of the Ising chain and the energy levels of the decorating unit. Therefore, they could calculate exact magnetic properties and theoretically show, for example, the existence of magnetization plateaus in certain bond-alternating chains.
The convenience of the decorated Ising chain as a theoretical model for spin chains derives from the relative ease with which exact solutions are obtained, in contrast for example with the pure Heisenberg chain, for which no exact partition function has been found. Up to now, this property of solvability has been the prime motive for the study of these chains in the literature. Indeed, in Ref. 3 the decorated Ising chain was considered as a substitute for the intractable Heisenberg model, and in Ref.4 the principle reason for introducing Ising bonds - to replace the more reasonable Heisenberg bonds - in a chain of $\mathrm{Cu}^{2+}$ ions was the desire to obtain a solvable model. This approach can be applied to any type of Heisenberg chain with a repeating unit: replace enough-preferably ferromagnetic - Heisenberg bonds by Ising bonds to obtain a decorated Ising chain that is solved easily and, in some cases, exhibits thermodynamic properties that are qualitatively comparable with those of the original chain. $[5]$

However, the role of the decorated Ising chain in the field of one-dimensional magnetism is not confined to that of a simplified model of realistic quantum spin chains. In this paper we show that some new molecular rings and chains are real examples of decorated Ising systems. Concretely, we treat a $[\mathrm{DyCuMoCu}]$ infinite chain ${ }^{7}$ and a $(\mathrm{DyCr})_{4}$ tetrameric ring. ${ }^{8}$ These compounds were recently synthesized in the course of the ongoing synthetic efforts to make new and better single-chain magnets (SCMs) and single-molecule magnets (SMMs), whose characteristic property is a blocking or slow relaxation of magnetization at low temperatures $[9$ We will not be concerned here with these dynamical aspects of their magnetism, but only with their static magnetic properties. A necessary property of SMMs and SCMs is a magnetic anisotropy. One line of approach is to introduce anisotropy by means of lanthanide ions, whether or not in combination with transition metal ions.10/11 The 


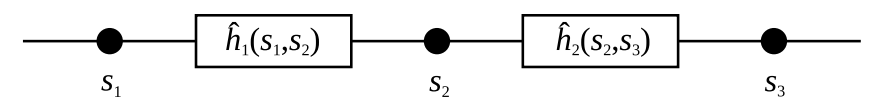

FIG. 2. Part of the decorated Ising chain. In each Ising bond is inserted an arbitrary statistical mechanical system ${ }^{1}$, called decorating unit, that interacts only with the two Ising spins $s_{i}$ at the vertices of the bond. The Ising spin variables commute with the Hamiltonian and have definite values in the eigenstates of the chain.

two compounds considered here are products of this approach, with dysprosium as lanthanide ion.

The $\mathrm{Dy}^{3+}$ ion plays a crucial role in these systems; the nature of the ground state of this ion in its ligand environment determines whether the system is a decorated Ising system or not and consequently, whether its partition function is exactly solvable or not. The ground Kramers doublet of $\mathrm{Dy}^{3+}$ must have complete uniaxial magnetic anisotropy $\sqrt{12}$ and must be separated from excited Kramers doublets by an amount that is large compared with the exchange coupling (typically, this separation must be $100 \mathrm{~cm}^{-1}$ or more) $! 3$ The required information on the ground and excited doublets of the $\mathrm{Dy}^{3+}$ monomer can be derived from ab initio calculations on the monomer complex, isolated from the polynuclear compound $\frac{78}{78}$

The $[\mathrm{DyCuMoCu}]$ chain and the $(\mathrm{DyCr})_{4}$ ring are shown to be decorated Ising chains in an arbitrarily directed magnetic field. The magnetic properties, in particular powder magnetization and susceptibility, are calculated with the help of the transfer-matrix method, which is a bit more general and convenient for numerical computation than the renormalization of the Ising parameters, which was used by Fisher and by Strečka and Jaščur. The results compare well with experiment and allow to determine values for the exchange coupling constants.

The excited crystal field Kramers doublets (or Stark levels) of $\mathrm{Dy}^{3+}$ are not included in the decorated Ising model. Because of their relatively low energetic position, these Kramers doublets can have a non-negligible contribution to the magnetic properties of the chain. We account for this in a first approximation by adding this contribution, as calculated ab initio for the monomeric $\mathrm{Dy}^{3+}$ complex, to the results of the decorated Ising model.

\section{THEORETICAL BACKGROUND}

\section{A. Decorated Ising chain and transfer-matrix solution}

The decorated Ising chain may be divided into units delimited by the Ising spins, as in Fig. 2. The Hamiltonian of the decorated Ising chain (or ring) of length $n$ may accordingly be written as follows:

$\hat{H}=\hat{h}_{1}\left(s_{1}, s_{2}\right)+\hat{h}_{2}\left(s_{2}, s_{3}\right)+\hat{h}_{3}\left(s_{3}, s_{4}\right)+\ldots+\hat{h}_{n}\left(s_{n}, s_{n+1}\right)$, where the subhamiltonians $\hat{h}_{i}$ correspond to units of the chain. If the chain is closed into a ring, periodic boundary conditions apply by identifying the last with the first spin: $s_{n+1} \equiv s_{1}$. The Ising spins $s_{i}$ commute with the subhamiltonians and the subhamiltonians commute with each other:

$$
\left[s_{i}, \hat{h}_{j}\right]=0 \quad \text { and } \quad\left[\hat{h}_{i}, \hat{h}_{j}\right]=0 .
$$

It is further assumed that there is no direct interaction between the decorating units themselves, i.e., two different $\hat{h}_{i}$ have no variables in common, except for an $s_{i}$ when they are neighbors. In writing Eq. (1) we made use of the fact that the Ising spins are conserved variables and may be considered as parameters rather than operators. In the case of spin- $1 / 2$, they take on the values $+1 / 2$ and $-1 / 2$, so that to each decorating unit there correspond four different subhamiltonians $\hat{h}_{i}\left(s_{i}, s_{i+1}\right)$ !14

Since there is no direct interaction between the decorating units of the chain, the subhamiltonians $\hat{h}_{i}$ in Eq. (1) work - for a given set of $s_{i}$ values - on disjunct spaces. An eigenfunction of $\hat{H}$ is then simply a direct product of $n$ independent eigenfunctions, one of each $\hat{h}_{i}$. The corresponding energy is:

$$
\begin{aligned}
E= & \varepsilon_{1 k_{1}}\left(s_{1}, s_{2}\right)+\varepsilon_{2 k_{2}}\left(s_{2}, s_{3}\right)+\varepsilon_{3 k_{3}}\left(s_{3}, s_{4}\right)+\ldots \\
& +\varepsilon_{n k_{n}}\left(s_{n}, s_{n+1}\right),
\end{aligned}
$$

where $\varepsilon_{i k_{i}}\left(s_{i}, s_{i+1}\right)$ is the $k_{i}$-th energy level of $\hat{h}_{i}\left(s_{i}, s_{i+1}\right)$.

Following Ref. 1 we write the partition function of one decorating unit for fixed $s_{i}$ and $s_{i+1}$ on the bond vertices:

$$
\psi_{i}\left(s_{i}, s_{i+1}\right)=\sum_{k} \exp \left[-\beta \varepsilon_{i k}\left(s_{i}, s_{i+1}\right)\right],
$$

where $\beta=1 / k T$. The total partition function for the chain is then given by

$$
\mathcal{Z}_{n}=\sum_{s_{1}, s_{2}, \ldots, s_{n+1}} \psi_{1}\left(s_{1}, s_{2}\right) \psi_{2}\left(s_{2}, s_{3}\right) \ldots \psi_{n}\left(s_{n}, s_{n+1}\right) .
$$

This is the well-known form of a transfer-matrix solution $\sqrt{15}$ Each decorated bond (or pair of neighboring Ising spins) is represented by a transfer matrix $T_{i}$ whose elements are the $\psi_{i}\left(s_{i}, s_{i+1}\right)$, the values of $s_{i}$ labeling the rows and the values of $s_{i+1}$ labeling the columns. Explicitly for the spin- $1 / 2$ decorated Ising chain:

$$
T_{i}=\left(\begin{array}{ll}
\psi_{i}\left(+\frac{1}{2},+\frac{1}{2}\right) & \psi_{i}\left(+\frac{1}{2},-\frac{1}{2}\right) \\
\psi_{i}\left(-\frac{1}{2},+\frac{1}{2}\right) & \psi_{i}\left(-\frac{1}{2},-\frac{1}{2}\right)
\end{array}\right) .
$$

Since most applications deal either with rings or very long chains, the boundaries can be identified $\left(s_{n+1} \equiv s_{1}\right)$ and expression (5) can be written as the trace of a matrix product:

$$
\mathcal{Z}_{n}=\operatorname{Tr}\left(T_{1} T_{2} \ldots T_{n}\right) .
$$

This is the most general expression for the partition function of a decorated Ising chain. If the chain has translational symmetry the partition function is expressed in 
terms of eigenvalues $\frac{[15]}{}$ Suppose that the chain is a repetition of identical decorated Ising bonds, then the $T_{i}$ are all the same, so that

$$
\mathcal{Z}_{n}=\operatorname{Tr}\left(T_{1}^{n}\right)=\sum_{i} \lambda_{i}^{n},
$$

where the $\lambda_{i}$ are the eigenvalues of $T_{1}$. According to Perron's theorem on positive matrices, the eigenvalue $\lambda_{0}$ with largest modulus is real, positive and nondegenerate ${ }^{16}$ This yields the simple but exact result for the free energy per unit cell of the infinite chain:

$$
f=-k T \lim _{n \rightarrow \infty} \frac{\ln \mathcal{Z}_{n}}{n}=-k T \ln \lambda_{0} .
$$

If a unit cell of the chain is spanned by $p$ decorated bonds instead of one then it is only necessary to combine $p$ transfer matrices into one new transfer matrix $\widetilde{T}=T_{1} T_{2} \ldots T_{p}$, with largest eigenvalue $\tilde{\lambda}_{0}$, to be used in Eqs. (8) and (9), where $n$ is to be replaced by $n / p$. This situation arises, for example, when the local easy axes on the Ising ions are not parallel but canted with respect to each other.

The solution in terms of transfer matrices, Eq. (7), is not limited to decorated spin-1/2 Ising chains. It is valid for chains having Ising spins of any multiplicity or a combination of Ising spins with different multiplicities, in which case the dimension of the transfer matrix is different from two by two. Another advantage of the transfer matrix method is that it can be readily extended to include next-nearest-neighbor bonds between the Ising spins. To this end, the transfer matrix has to be enlarged so that it does not jump from one Ising spin to the next, but from one pair of Ising spins to the next pair $\frac{15}{15}$

\section{B. $\mathrm{Dy}^{3+}$ as Ising spin}

We are interested in this paper in molecular chains or rings that can be described by a decorated Ising model. This means that their low-energy spectrum can be modeled to satisfactory accuracy by an effective Hamiltonian that has the properties described in Section IIA. It has been noted there that the composition of the decorating unit is basically arbitrary and we need therefore not consider the properties of that part. Instead, our attention goes here to the molecular realization of the Ising spin $\left[s_{i}\right.$ in Eq. [1]]. We focus on $\mathrm{Dy}^{3+}$ because this ion is used in the two examples studied below, but the discussion applies equally well to several other trivalent lanthanides and also to some transition metal ions.

It is known that lanthanide ions in a coordination environment are often well described by crystal field theory applied to the ground $|L S J\rangle$ level. One assumes that $J, L$, and $S$ remain good quantum numbers. $\mathrm{Dy}^{3+}$ is a Kramers ion that belongs to the second half of the lanthanide series, whose ground level is ${ }^{6} \mathrm{H}_{15 / 2}\left(f^{9}\right)$, with associated Landé factor $g=4 / 3$. This multiplet splits into eight Kramers doublets by the crystal field perturbation (except for high-symmetric environments belonging to the $T, O$, or $K$ point groups, which split the multiplet in less than eight levels). It will be useful to view each Kramers doublet as an effective spin- $1 / 2$ with its own $g$ factors (3 in number) and corresponding magnetic axes. For example, take the Kramers doublet $\left|M_{J}= \pm 15 / 2\right\rangle$ (of the ${ }^{6} \mathrm{H}_{15 / 2}$ level), quantized with respect to the $z$ axis. Its $g$ factors are $g_{z}=2(4 / 3)(15 / 2)=20$ and $g_{x}=g_{y}=0$.

If the action of the crystal field on $\mathrm{Dy}^{3+}$ is such that the lowest Kramers doublet is separated from the next one by an energy that is large compared to the energy of interaction with the magnetic field and the exchange interaction with neighboring ions, we can omit all excited Kramers doublets from the Hamiltonian and keep only the lowest doublet. In this way $\mathrm{Dy}^{3+}$ is described by a spin of $1 / 2$ and every interaction in which it takes part enters the Hamiltonian as a linear combination of the three spin operators $s_{x}, s_{y}$, and $s_{z} \cdot \frac{17]}{\text { If }}$ we now want $\mathrm{Dy}^{3+}$ to be an Ising spin, defined by the first commutation relation in Eq. (2), it is clear that only one of these spin components, say $s_{z}$ or simply $s$, may actually appear in the Hamiltonian. In other words, there must be no interaction that creates an off-diagonal matrix element between the two components of the Kramers doublet. This can be shown to be true with high accuracy if the lowest Kramers doublet is $\left|M_{J}= \pm 15 / 2\right\rangle$.

The two interactions of importance here are the Zeeman interaction with the magnetic field and the exchange interaction with other magnetic ions. The Zeeman Hamiltonian follows directly from the $g$ factors of the Kramers doublet (vide supra), and is

$$
-\mu_{\mathrm{B}} g_{z} s_{z} B_{z},
$$

where $B_{z}$ is the $z$ component of the applied magnetic field. Note that the field may be applied in any direction, but it is only the $z$ component that interacts with the Kramers doublet because $g_{x}=g_{y}=0$. The vanishing of $g_{x}$ and $g_{y}$ in $\left|M_{J}= \pm 15 / 2\right\rangle$ follows from the selection rule stating that a vector operator (the magnetic moment in this case) cannot connect states for which $M_{J}$ differs by more than one unit. We will sometimes refer to the $z$ axis as the anisotropy axis, to stress that it is the only magnetic axis with nonvanishing $g$ factor.

To evaluate the effect of exchange interaction, we must first take a closer look at the composition of the Kramers doublet. In terms of the Russel-Saunders states $\left|M_{S}\right\rangle\left|M_{L}\right\rangle$ we have

$$
\begin{aligned}
& |+15 / 2\rangle=|+5 / 2\rangle|+5\rangle \\
& |-15 / 2\rangle=|-5 / 2\rangle|-5\rangle .
\end{aligned}
$$

In a basic (super)exchange process between two magnetic centers, one electron of each center takes part. If we look at one center, the process removes an electron with certain spin projection (up or down) and puts it 
back on the center either with the same or with opposite spin projection 18 This gives rise to the selection rule $\Delta M_{S}=0, \pm 1$. If the exchange interaction is to connect both components of the Kramers doublet in Eq. (11), at least five successive processes are needed, for $\Delta M_{S}= \pm 5$. In other words, not one but five electron spins have to be flipped to connect $|+15 / 2\rangle$ with $|-15 / 2\rangle$. If the basic exchange process (i.e. the one for which $\Delta M_{S}=0, \pm 1$ ) occurs in, say, $k$ th order ${ }^{19}$ of perturbation theory then an off-diagonal matrix element between the two components can only appear in $(5 k)$ th order of perturbation theory. It is therefore reasonable to assume that the offdiagonal matrix element is negligibly small compared to the diagonal matrix elements $\left(\Delta M_{S}=0\right)$ so that the effect of exchange interaction on the Kramers doublet is accurately described by the $s_{z}$ spin operator only.

Note that we derived the selection rule for exchange interaction on the basis of the spin quantum number $M_{S}$ only, without paying attention to the angular momentum quantum number $M_{L}$, although $M_{L}$ changes even more than $M_{S}$ between the states of the Kramers doublet (11). The existence and precise form of a selection rule for $M_{L}$ depends on the spatial symmetry of the exchange problem under consideration. $\Delta M_{L}$ is therefore not as useful as $\Delta M_{S}$ for predicting the vanishing of certain matrix elements of exchange interaction. Note however that, even in the lowest symmetry, there is a maximum to the amount that $M_{L}$ can change in the basic exchange process described in the previous paragraph: within the $f$ orbitals, a one-electron process can bring about at most a change of $\Delta M_{L}= \pm 6$. So we would have, in general, that a basic exchange process can induce the following changes in a lanthanide state:

$$
\begin{aligned}
& \Delta M_{S}=0, \pm 1 \\
& \left|\Delta M_{L}\right| \leq 6 .
\end{aligned}
$$

Thus at least two steps of this kind are needed to bridge $\Delta M_{L}= \pm 10$, but at least five are needed to bridge $\Delta M_{S}= \pm 5$. So in this case, the selection rule of $M_{S}$ gives the stronger result and leads to the conclusionsreached in the last paragraph - on the matrix elements of exchange interaction in the Kramers doublet (11).

We can now derive the precise form of that part of the effective Hamiltonian that refers to the exchange interaction between a $\mathrm{Dy}^{3+}$ ion [with ground state [11)] and another magnetic center. We consider two cases, which we shall encounter in the examples in Sections [III and IV in the first case the other center is another Dy ${ }^{3+}$ ion; in the second case the other center is an ion with an isotropic spin moment $\mathbf{S}$. Consider first the exchange interaction with another $\mathrm{Dy}^{3+}$ ion. We assume that the second $\mathrm{Dy}^{3+}$ has the same property of uniaxiality as the first one and that it also shares all other relevant properties discussed in the previous paragraphs. Then both ions are represented by a spin- $1 / 2$ doublet with a local anisotropy axis $\hat{\mathbf{z}}_{i}(i=1,2)$ and we already know that the effect of exchange interaction in each doublet is proportional to $s_{z_{i}}$. Therefore the exchange Hamiltonian is necessarily of the form

$$
-J s_{z_{1}} s_{z_{2}}
$$

where it is understood that the first spin belongs to ion 1 and the second to ion 2 . Note that $\hat{\mathbf{z}}_{1}$ and $\hat{\mathbf{z}}_{2}$ need not be parallel with each other.

As a second case, consider the interaction between Dy $^{3+}$ (anisotropy axis $\hat{\mathbf{z}}_{1}$ ) and an isotropic spin $\mathbf{S}_{2}$. The latter may typically be a transition metal ion with quenched orbital momentum. We found that the exchange processes that contribute do not change the spin projection on the $\mathrm{Dy}^{3+}$ ion $\left(\Delta M_{S_{1}}=0\right.$, quantization axis $\hat{\mathbf{z}}_{1}$ ), and that this result is independent of the exchange partner. It is also known that every exchange process commutes with the total spin (the matrix elements involved are spin-independent matrix elements of kinetic, potential, and Coulomb energy $\sqrt{18}$ ), so that $\Delta M_{S_{1}}+\Delta M_{S_{2}}=0$. It follows then that $\Delta M_{S_{2}}=0$, or, the exchange Hamiltonian commutes with the $z_{1}$ component of $\mathbf{S}_{2}$. The simplest expression compatible with this requirement is

$$
-J s_{z_{1}} S_{z_{1}}
$$

where $s$ naturally represents $\mathrm{Dy}^{3+}$ and $S$ represents $\mathbf{S}_{2}$. The interaction is of Ising form with the anisotropy axis of $\mathrm{Dy}^{3+}$ as Ising axis. Note that, when $S_{2}>1 / 2$, higher powers of $S_{z_{1}}$ may enter the Hamiltonian. Considering exchange interaction as a perturbation however, one can usually assume that the lowest-order contribution, Eq. (14), is the leading term.

There are other cases conceivable, for example dipoledipole interaction between the moment of $\mathrm{Dy}^{3+}$ and a neighboring moment. One will always find, as above, that the Hamiltonian is a product of $s_{z}$ (belonging to $\mathrm{Dy}^{3+}$ ) and a part that belongs to the other ion and whose form depends on the kind of the other ion and on the details of the interaction.

We have now obtained that a $\mathrm{Dy}^{3+}$ ion, if its ground state is $\left|M_{J}= \pm 15 / 2\right\rangle$, fairly well separated from excited states, interacts with the magnetic field and with neighboring ions as an Ising spin-1/2, in the sense that the interaction is always proportional to $s_{z}$, as expressed in the Eqs. 10, (13), and (14). This means that a chain-like molecular structure having $\mathrm{Dy}^{3+}$ ions of this kind at regular positions in the chain would meet the requirements of a decorated Ising chain, given in part by Eqs. (1) and (2). It remains of course to be shown that $\left|M_{J}= \pm 15 / 2\right\rangle$ can indeed be the ground state of a coordinated Dy ${ }^{3+}$ ion in a polynuclear complex.

At first sight, this seems rather unlikely. $\mid M_{J}=$ $\pm 15 / 2\rangle$ is an eigenstate of cylindrical symmetry. Within lanthanide $f^{n}$ states, the crystal field is effectively cylindrical if there is, at least, an eightfold rotation axis $\left(C_{8}\right)$ or rotation-inversion axis $\left(S_{8}\right) ! 20 \quad S_{8}$ symmetry has been obtained, for example, in mononuclear bis(phtalocyaninato) sandwich complexes of the lanthanides.21 Even when such high symmetry is at- 
tained, the ground state is not necessarily the cylindrical doublet with highest $\left|M_{J}\right|$ value. ${ }^{21}$ Apart from that, the symmetry of the coordination sphere of a lanthanide ion in a polynuclear, possibly heterometallic, complex or chain is usually much lower or even completely absent. Such is the case for the two examples considered in this paper. On the basis of symmetry alone, there is thus no reason to expect $\left|M_{J}= \pm 15 / 2\right\rangle$ to be an eigenstate, let alone the ground state. Nevertheless, recent $a b$ initio calculations have revealed the unexpected result that the ground state of several low-symmetry complexes of $\mathrm{Dy}^{3+}$ is very close to $\left.\left|M_{J}= \pm 15 / 2\right\rangle\right\rangle^{[7 / 8|22|}[24$ They used the multiconfigurational, wavefunction-based CASSCF/RASSI-SO method, to obtain accurate wavefunctions for several of the lowest Kramers doublets. Calculation of the principal $g$ factors of these states gives an indication of their composition. It was found in several cases that the ground doublet has $g_{z}$ close to, but lower than 20, and $g_{x}$ and $g_{y}$ close to 0 . This corresponds to a doublet mainly composed of $\left|M_{J}= \pm 15 / 2\right\rangle$.

With evidence of $a b$ initio calculations it is thus possible to identify $\mathrm{Dy}^{3+}$ in certain coordination environments as an Ising spin-1/2 (to a good approximation). We will use this information to identify the compounds in Sections III and IV as decorated Ising chains (or rings).

To conclude this section we remark that one cannot deduce from the vanishing of two $g$ factors alone that a Kramers doublet will behave as an Ising spin. It will, of course, in its interaction with the magnetic field [Eq. (10]], but this will not, in general, be true for the exchange interaction. Take again the example of $\mathrm{Dy}^{3+}$, supposing the ground state is the Kramers doublet $\left|M_{J}= \pm 7 / 2\right\rangle$. It is perfectly uniaxial because $g_{x}=g_{y}=0$ and $g_{z}=9 \frac{1}{3}$. A closer look at the expansion of $\left|M_{J}= \pm 7 / 2\right\rangle$ in terms of the Russel-Saunders states $\left|M_{L}\right\rangle\left|M_{S}\right\rangle$ shows, however, that the selection rules in Eq. 12 permit a matrix element to exist between $|+7 / 2\rangle$ and $|-7 / 2\rangle$, therefore introducing non-Ising terms (i.e., $s_{x}$ and $s_{y}$ ) in the same order of perturbation theory as the Ising term in the exchange Hamiltonian.

\section{Nature of the eigenstates and level crossings}

There exists a similarity between the spectra of the decorated and undecorated Ising chains at which we want to take a closer look here. We suppose infinite, periodic chains, or periodic, even-membered rings (In odd-membered rings spin-frustration complicates the picture). We also limit ourselves to chains with Ising spins of $1 / 2$.

The undecorated, or simple, Ising chain in a magnetic field $B$ parallel with the $z$ axis is given by Eq. (1) and

$$
\hat{h}_{i}=-J s_{i} s_{i+1}-\mu_{\mathrm{B}} g s_{i} B,
$$

where $s_{i}$ is the $z$ component of $\mathbf{s}_{i}\left(s_{i}= \pm 1 / 2\right)$. We assume, without loss of generality, $B \geq 0$. The eigenstates of the chain are spin configurations like ( $\uparrow \downarrow \uparrow \downarrow \ldots)$, etc.
Of the $2^{n}$ eigenstates only two distinct ones can be the ground state: the ferromagnetic $(\mathrm{F})$ and the antiferromagnetic (AF):

$$
\begin{aligned}
\mathrm{F}:(\uparrow \uparrow \uparrow \uparrow \ldots) & \text { if } J>0 \text { or } J<0 \text { and } \mu_{\mathrm{B}} g B>|J| \text {, } \\
\mathrm{AF}:(\uparrow \downarrow \uparrow \downarrow \ldots) & \text { if } J<0 \text { and } \mu_{\mathrm{B}} g B<|J| .
\end{aligned}
$$

When $B=0$ time-reversal symmetry makes every state degenerate with the state formed by flipping all the spins. This degeneracy is meant to be implied in $(16)$, where only one of two states is shown in each case. Only the two AF states remain degenerate when $B \neq 0$. When $J<0$ a ground state level crossing occurs from AF to $\mathrm{F}$ when $B$ is increased. At the point of crossing $\left(\mu_{\mathrm{B}} g B=|J|\right)$ the two AF states are degenerate together with all states derived from an AF state by flipping one or more downspins up. However, no other state than AF or $\mathrm{F}$ can be the ground state at any other value of $B$. Thus the ground state of the Ising chain is either $\mathrm{F}$ or AF, and they are degenerate, together with an infinite number of other states, at the crossing point.

We now decorate the Ising bonds with identical but arbitrary units to obtain a periodic decorated Ising chain. The spectrum is given by Eq. (3). Since the chain is periodic, the spectrum of the individual units $\hat{h}_{i}$ is independent of $i$ and the energies may be written as $\varepsilon_{k}$, with $k$ ranging over the eigenstates of $\hat{h}_{i}$. There are four sets of $\varepsilon_{k}: \varepsilon_{k}(\uparrow \uparrow), \varepsilon_{k}(\uparrow \downarrow), \varepsilon_{k}(\downarrow \uparrow)$, and $\varepsilon_{k}(\downarrow \downarrow)$, in an obvious notation. Notice that the eigenstates of this chain can still be classified according to the configuration of the Ising spins: $(\uparrow \uparrow \downarrow \uparrow \downarrow .$.$) etc., which follows from the fact that$ all the $s_{i}$ and $\hat{H}$ form a commuting set of observables.

An interesting question is whether the same rules hold for the ground state of the decorated Ising chain as did for the simple Ising chain. The answer is yes; the ground state is either F or AF (referring to the Ising spin configuration) and a crossing between them is possible, with the same number and kind of degenerate states as in the simple Ising chain. To show this, we have to consider only the lowest eigenstate belonging to each of the $2^{n}$ possible Ising spin configurations. In these states every unit is in its lowest possible state for the given orientation of the neighboring Ising spins: $\varepsilon_{1}\left(s, s^{\prime}\right)$ (we assume this energy to be nondegenerate), so that the total energy of the chain state is

$$
E=n_{\uparrow \uparrow} \varepsilon_{1}(\uparrow \uparrow)+n_{\downarrow \downarrow} \varepsilon_{1}(\downarrow \downarrow)+n_{\downarrow \uparrow} \varepsilon_{1}(\downarrow \uparrow)+n_{\uparrow \downarrow} \varepsilon_{1}(\uparrow \downarrow),
$$

where $n_{\uparrow \uparrow}$ denotes the number of pairs of neighboring Ising spins that are both spin up, etc. For example, in the $\mathrm{F}$ configuration in Eq. (16), $n_{\uparrow \uparrow}=n$ (periodic boundary conditions are assumed), while in the AF configuration, $n_{\uparrow \uparrow}=0$. The eigenstates we have just described, with energy (17), are in an obvious one-to-one correspondence with the eigenstates of the simple Ising chain. The ground state is found by minimizing 17 with respect to 
the $n_{s s^{\prime}}$, under the restrictions

$$
\begin{aligned}
n_{\uparrow \uparrow}+n_{\downarrow \downarrow}+n_{\downarrow \uparrow}+n_{\uparrow \downarrow} & =n \\
n_{\downarrow \uparrow} & =n_{\uparrow \downarrow} .
\end{aligned}
$$

The first relation states that the total number of Ising spins (or, equivalently, unit cells) is $n$. The second relation follows from the fact that, in a cyclic spin configuration, every $\downarrow \uparrow$ pair must eventually be followed by a $\uparrow \downarrow$ pair, possibly after a number of $\uparrow \uparrow$ pairs. Another restriction is that whenever both $n_{\uparrow \uparrow}$ and $n_{\downarrow \downarrow}$ are not zero, $n_{\downarrow \uparrow}$ [and by Eq. (18) also $n_{\uparrow \downarrow}$ ] must be at least one. Using 18. we can rewrite Eq. 17) as

$$
\begin{aligned}
E & =n_{\uparrow \uparrow}\left(\varepsilon_{1}(\uparrow \uparrow)-\frac{1}{2}\left[\varepsilon_{1}(\uparrow \downarrow)+\varepsilon_{1}(\downarrow \uparrow)\right]\right) \\
& +n_{\downarrow \downarrow}\left(\varepsilon_{1}(\downarrow \downarrow)-\frac{1}{2}\left[\varepsilon_{1}(\uparrow \downarrow)+\varepsilon_{1}(\downarrow \uparrow)\right]\right) \\
& +\frac{n}{2}\left[\varepsilon_{1}(\uparrow \downarrow)+\varepsilon_{1}(\downarrow \uparrow)\right],
\end{aligned}
$$

where we see that only the average $\left[\varepsilon_{1}(\uparrow \downarrow)+\varepsilon_{1}(\downarrow \uparrow)\right] / 2$ of the "antiparallel" energies enters the equation. The last term is a constant and can be discarded for the purpose of relative energy considerations.

We can now derive the values of $n_{\uparrow \uparrow}$ and $n_{\downarrow \downarrow}$ for the ground state of the chain, keeping in mind that $\varepsilon_{1}\left(s, s^{\prime}\right)$ is a function of the magnetic field $\mathbf{B}$. Suppose then, first, that $\mathbf{B}=0$. Time reversal symmetry asserts that $\varepsilon_{1}(\uparrow \uparrow)=\varepsilon_{1}(\downarrow \downarrow)$ and $\varepsilon_{1}(\uparrow \downarrow)=\varepsilon_{1}(\downarrow \uparrow)$. It is simple to see that, depending on the relative ordering of $\varepsilon_{1}(\uparrow \uparrow)$ and $\varepsilon_{1}(\uparrow \downarrow), E$ is minimal in the $\mathrm{F}$ configuration $\left(n_{\uparrow \uparrow}=n\right.$ or $\left.n_{\downarrow \downarrow}=n ; n_{\uparrow \downarrow}=n_{\downarrow \uparrow}=0\right)$ when $\varepsilon_{1}(\uparrow \uparrow)<\varepsilon_{1}(\uparrow \downarrow)$ or in the AF configuration $\left(n_{\uparrow \uparrow}=n_{\downarrow \downarrow}=0 ; n_{\uparrow \downarrow}=n_{\downarrow \uparrow}=n / 2\right)$ when $\varepsilon_{1}(\uparrow \uparrow)>\varepsilon_{1}(\uparrow \downarrow)$ (we exclude the possibility of equality of both energies from the discussion; $\varepsilon_{1}(\uparrow \uparrow)=\varepsilon_{1}(\uparrow \downarrow)$ would correspond, in the simple Ising chain 15 , with $J=0)$. When $\mathbf{B} \neq 0$, time reversal symmetry is not operative, and we have, in general, four different energies $\varepsilon_{1}\left(s, s^{\prime}\right)$. The equation (19) shows that the configuration that minimizes $E$ is determined by the sign of the two terms in round brackets; if both are positive, then $n_{\uparrow \uparrow}=n_{\downarrow \downarrow}=0$ (AF configuration); if at least one of them is negative, then either $n_{\uparrow \uparrow}=n$ or $n_{\downarrow \downarrow}=n$ (F configuration), depending on whether respectively $\varepsilon_{1}(\uparrow \uparrow)$ or $\varepsilon_{1}(\downarrow \downarrow)$ is lower.

Finally, the magnetic field can induce a transition from the AF to an $\mathrm{F}$ ground state configuration, say with all Ising spins up. This happens when

$$
\varepsilon_{1}(\uparrow \uparrow)=\frac{1}{2}\left[\varepsilon_{1}(\uparrow \downarrow)+\varepsilon_{1}(\downarrow \uparrow)\right],
$$

and $\varepsilon_{1}(\uparrow \uparrow)<\varepsilon_{1}(\downarrow \downarrow)$. At this point, the ground state configurations are all those for which $\left\{n_{\downarrow \downarrow}=0, n_{\uparrow \uparrow}=\right.$ $0,2,4, \ldots, n\}$, exactly the same as in the simple Ising chain.

We find thus a complete analogy between the simple and the decorated Ising chain as far as the ground state Ising spin configuration is concerned. The only possible configurations are the fully antiferromagnetically aligned and the fully ferromagnetically aligned configurations. No "intermediate" configuration can be the ground state. The only exception is the crossing point between $\mathrm{AF}$ and $\mathrm{F}$, where there is a high degeneracy of configurations. These conclusions are independent of the nature of the decorating unit.

Although the decorated Ising model predicts that the $\mathrm{AF}$ and $\mathrm{F}$ ground states are both doubly degenerate (in zero field), this degeneracy is not a result of the spatial symmetry: in the cyclic group $C_{n}$, the two AF components combine into irreducible representations (irreps) $A$ and $B$, while the two $\mathrm{F}$ components transform as two $A$ irreps. Introduction of neglected terms in the Hamiltonian, that destroy the Ising property, could split these ground state components.

The decorated Ising chain affords two new kinds of ground state level crossings, not present in the simple Ising chain. The first of these is the transition between one $\mathrm{F}$ configuration of the Ising spins and the other: $(\uparrow \uparrow \uparrow \uparrow, \ldots) \leftrightarrow(\downarrow \downarrow \downarrow \downarrow, \ldots)$. This transition takes place when $\varepsilon_{1}(\uparrow \uparrow)=\varepsilon_{1}(\downarrow \downarrow)$. This level crossing, induced by the magnetic field, can for example be encountered in frustrated Ising-Heisenberg chains. ${ }^{25}$ A second kind of new ground state transition arises from the crossing of levels within a decorating unit. A ground state crossing can result in which the Ising spin configuration remains the same but the state corresponding to $\varepsilon_{1}\left(s, s^{\prime}\right)$ crosses with the state corresponding to $\varepsilon_{2}\left(s, s^{\prime}\right)$. More precisely this happens in the F configuration when $\varepsilon_{1}(\uparrow \uparrow)=\varepsilon_{2}(\uparrow \uparrow)$ and in the AF configuration when either $\varepsilon_{1}(\uparrow \downarrow)=\varepsilon_{2}(\uparrow \downarrow)$ or $\varepsilon_{1}(\downarrow \uparrow)=\varepsilon_{2}(\downarrow \uparrow)$, or both. The previous paragraphs have shown that we do not need to consider configurations other than $\mathrm{F}$ and $\mathrm{AF}$ for the ground state.

Level crossings are usually connected with the presence of good quantum numbers. For the Ising-type crossings, the relevant conserved quantities are the $n$ Ising spins $\left\{s_{i}\right\}$. The crossing of energy levels within the decorating unit should be associated with a conserved variable that is internal to that unit, much the same as in isolated molecules. In Section III we will encounter an example where both transitions - Ising type and internal typeoccur in a magnetic field.

\section{Magnetization of powder samples}

In the following sections we will be comparing our theoretical results with measurements performed on powder samples of the crystalline compounds. In this section we consider the powder averaging of magnetization for the example of the simple Ising chain.

Let $\theta$ and $\phi$ be the polar angles of the magnetic field vector with respect to a molecular reference frame, then the free energy is a function of $\theta, \phi$, and the strength of the field, $B: f(\theta, \phi, B)$. The projection of the magneti- 
zation on the field direction $\hat{\mathbf{e}}_{B}$ is

$$
\hat{\mathbf{e}}_{B} \cdot \mathbf{M}(\theta, \phi, B)=-\frac{\partial f(\theta, \phi, B)}{\partial B} .
$$

Averaging over one hemisphere gives the powder magnetization

$$
M(B)=\frac{1}{2 \pi} \int_{0}^{2 \pi} \int_{0}^{\pi / 2}-\frac{\partial f(\theta, \phi, B)}{\partial B} \sin \theta d \theta d \phi
$$

Let us see how the powder averaging affects the magnetization curve for a simple Ising chain. Take a spin- $1 / 2$ infinite antiferromagnetic Ising chain with anisotropy axes parallel with each other and with the $z$ axis, and uniaxial $g$-factors $\left(g_{x}=g_{y}=0\right.$ and $\left.g_{z} \equiv g\right)$. This could for example be realized by a chain of identical $\mathrm{Dy}^{3+}$ units (see Section IIB). The Hamiltonian is given by Eq. (1), substituting

$$
\hat{h}_{i}=-J s_{i} s_{i+1}-\mu_{\mathrm{B}} g s_{i} B \cos \theta,
$$

where $B \cos \theta$ is the $z$ component of the magnetic field, and $J<0$. Defining $j \equiv J / k$ and $b \equiv \mu_{\mathrm{B}} g B / k$, the magnetization, which has only a nonzero $z$ component, is 15

$$
M_{z}=\frac{\mu_{\mathrm{B}} g}{2} \frac{\sinh [b \cos \theta / 2 T]}{\sqrt{\sinh ^{2}[b \cos \theta / 2 T]+e^{-j / T}}} .
$$

The projection on the field direction [Eq. (21)] is $M_{z} \cos \theta$. Plugging this in Eq. 22 and substituting $u=\cos \theta$ yields the powder magnetization of the Ising chain

$$
M=\frac{\mu_{\mathrm{B}} g}{2} \int_{0}^{1} \frac{\sinh [b u / 2 T]}{\sqrt{\sinh ^{2}[b u / 2 T]+e^{-j / T}}} u d u .
$$

Because the Hamiltonian in Eq. (23) does not depend on $\phi$, this variable has been integrated out in Eq. 24).

Figs. 3 and 4 show plots of magnetization versus magnetic field, for coupling constant $j=-1 \mathrm{~K}$. The step-like appearance of $M_{z}$ is associated with the ground state crossing that occurs at $b \cos \theta=|j|$. At that point, the antiferromagnetic ground state (or rather ground state Ising doublet) is replaced by the ferromagnetic state (all spins up). Consequently, the magnetization jumps from zero to the saturation value of 0.5 , as seen in Fig. 3 . The magnetization of a powder sample of the same Ising chain is shown in Fig. 4. Before the crossing point, $M$ behaves qualitatively the same as $M_{z}$. After the crossing point however, $M$ is seen to reach only slowly its saturation value, which is half of the saturation value of $M_{z}$, viz. 0.25 . The limiting curve of $M$ as $T \rightarrow 0 \mathrm{~K}$ can be calculated exactly from Eq. 24:

$$
\left.M\right|_{T \rightarrow 0}= \begin{cases}0 & \text { if } b \leq|j| \\ \frac{\mu_{\mathrm{B}} g}{4}\left(1-\frac{|j|^{2}}{b^{2}}\right) & \text { if } b>|j| .\end{cases}
$$

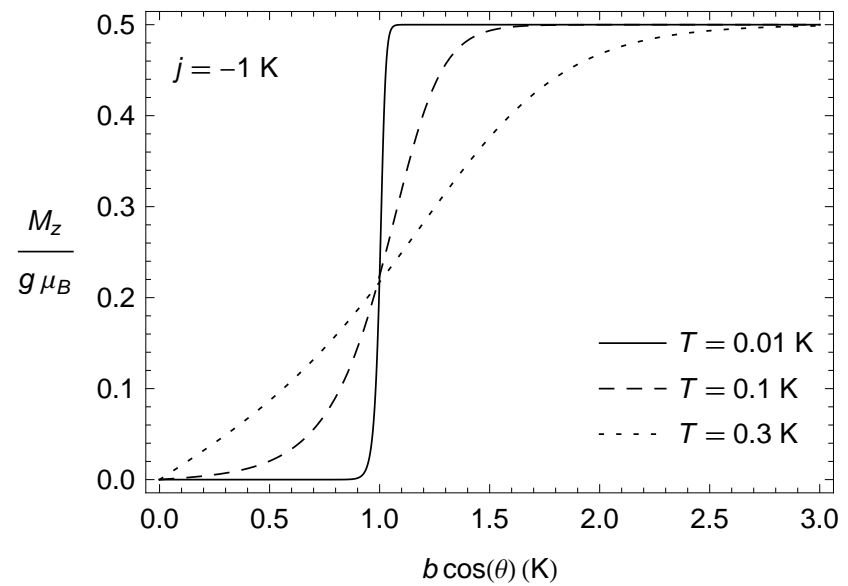

FIG. 3. Magnetization along the $z$ direction versus field of the antiferromagnetic Ising chain, defined in Eq. (23). The curve approaches a perfect step as $T \rightarrow 0 \mathrm{~K}$.

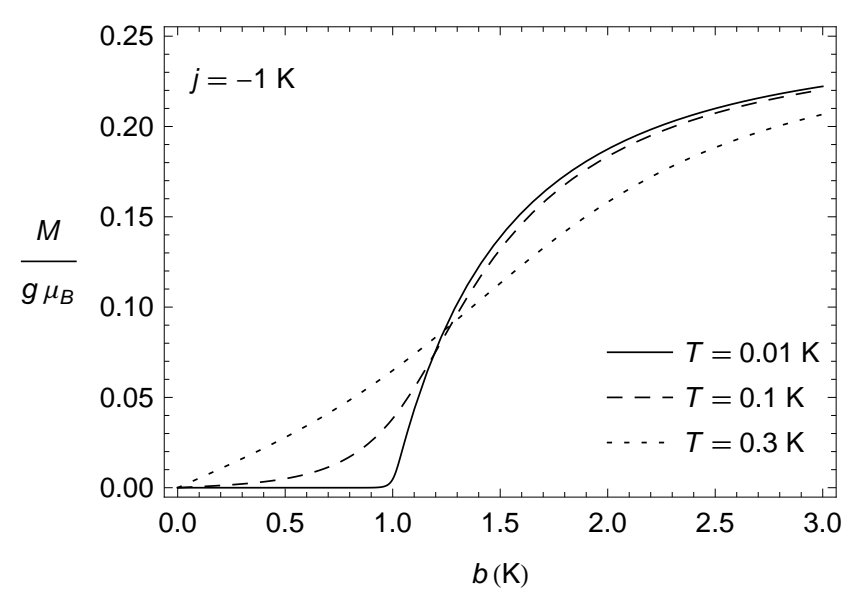

FIG. 4. Magnetization of a powder sample of antiferromagnetic Ising chains. The low-temperature limiting curve is given in Eq. (25).

(This is of course only valid for the antiferromagnetic case $j<0$.) In Fig. 4 this limiting curve is very closely approximated by the curve at $T=0.01 \mathrm{~K}$. Clearly, the sharp step of $M_{z}$ transforms in the powder to the concave form displayed by $M$. This is understood from the fact that, in a powder, for a given field $b>|j|$, there is always a fraction of molecules that is not magnetized (in the sense that they are in the antiferromagnetic ground state) because they are oriented so with respect to the field, that $b \cos \theta<|j|$ (see Fig. 33). The powder saturates only when every molecule is fully magnetized, and this happens only for $b \rightarrow \infty$. Therefore, $M$ (at $0 \mathrm{~K}$ ) does not abruptly saturate at the crossover point, but increases slowly to saturation. 


\section{E. Corrections for the contribution of excited Kramers doublets}

In deriving the exchange Hamiltonian in Section IIB we assumed that only the lowest Kramers doublet on Dy ${ }^{3+}$ took part. This is certainly a good approximation when the gap between the lowest and the second-lowest Kramers doublet is much larger then the strength of the exchange interaction. However, the excited Kramers doublets often have to be taken into account to a certain degree of approximation if a comparison with experimental data on susceptibility and magnetization is desired. The crystal field splitting of the ${ }^{6} \mathrm{H}_{15 / 2}$ level is of the order of $k T$ at room temperature. This gives rise to two effects: (i) a thermal population of excited Kramers doublets, and (ii) a modification of the lowest Kramers doublet as a function of the applied magnetic field by interaction with the excited doublets.

Effect (i) is mainly visible in the temperature dependence of $\chi T$, where $\chi$ is the powder magnetic susceptibility: for a single $\mathrm{Dy}^{3+}$ center, $\chi T$ increases monotonically with increasing temperature, from the value of the ground doublet at $0 \mathrm{~K}$ to the saturation value of ${ }^{6} \mathrm{H}_{15 / 2}$ at higher temperatures. Effect (ii) gives rise to temperatureindependent paramagnetism (TIP). It is visible at temperatures sufficiently low so that only the ground doublet is occupied. It contributes a linear increase of $\chi T$ with $T$ and a linear increase of the magnetization $M$ with the applied field $B$.

In the simplest approximation, the contribution of the excited Kramers doublets to the magnetic properties of the chain is equal to the contribution they have to the properties of the single, isolated $\mathrm{Dy}^{3+}$ ion in the same ligand environment it has in the chain. Let $\chi_{\text {DIC }}$ and $M_{\text {DIC }}$ denote susceptibility and magnetization derived from the decorated Ising chain model, and let $\chi_{\text {Dy }}$ denote the susceptibility of the $\mathrm{Dy}^{3+}$ center and $\mu_{\mathrm{Dy}}^{\prime} B$ the magnetic moment induced by $B$ in the ground doublet of the Dy ${ }^{3+}$ center, then the corrected properties are (supposing one $\mathrm{Dy}^{3+}$ ion per unit cell)

$$
\begin{aligned}
\chi T & =\chi_{\mathrm{DIC}} T+\left(\chi_{\mathrm{Dy}} T-\left.\chi_{\mathrm{Dy}} T\right|_{T=0}\right), \\
M & =M_{\mathrm{DIC}}+\mu_{\mathrm{Dy}}^{\prime} B .
\end{aligned}
$$

The last equation assumes that only the ground doublet of $\mathrm{Dy}^{3+}$ is occupied. This is correct at the temperature at which magnetization curves are usually recorded (e.g., $2 \mathrm{~K}$ ). If necessary, corrections due to other magnetic ions can be added in the same way. $\chi_{\mathrm{Dy}}$ and $\mu_{\text {Dy }}^{\prime}$ can be obtained from multiconfigurational $a b$ initio calculations ${ }^{78 \mid 22123}$, or, in oligonuclear complexes, experimentally by replacing certain magnetic ions with diamagnetic ions. 26

The equations (26) are evidently correct in the limit of vanishing exchange interactions. The assumption we make is that the exchange interactions are sufficiently small for these corrections to remain valid. A more ac-

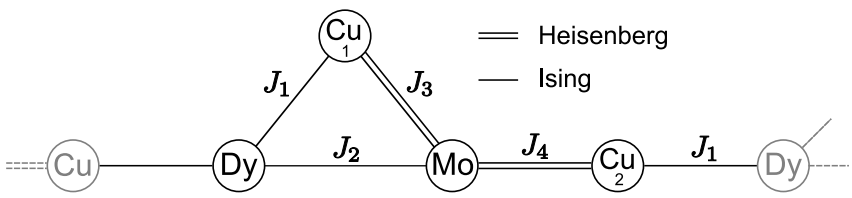

FIG. 5. Scheme of one unit cell (black) of the $[\mathrm{DyCuMoCu}]_{\infty}$ chain, showing type of exchange interactions and labeling of exchange constants. See Ref. [7] for the complete molecular structure.

curate approach should take into consideration the fact that the excited Kramers doublets also participate in the exchange interaction with neighbors. This is however out of the scope of the decorated Ising model.

\section{III. [DyCuMoCu $]_{\infty}$ POLYMER AS ISING-HEISENBERG CHAIN}

We now turn our attention to two actual examples of decorated Ising models based on $\mathrm{Dy}^{3+}$ : a chain, treated in this section, and a four-ring treated in the following section. The problem is approached as follows: the Hamiltonian for the chain in a magnetic field is formulated, with the help of the considerations in Section IIB. Values of the $g$ factors of the magnetic ions are taken directly from $a b$ initio calculations, reported elsewhere. ${ }^{78}$ This leaves the exchange coupling constants as parameters of the model, to be fitted by comparison with experimental magnetization and susceptibility data. (In Section IV the direction of the anisotropy axis contributes one extra parameter.)

The $[\mathrm{DyCuMoCu}]$ chain was recently synthesized and details of its chemical composition and structure are given in Ref. 7. The crystal structure was found to consist of parallel linear chains each made of [DyCuMoCu] unit cells. Fig. 5 shows how the metal ions are connected by ligand bridges. Multiconfigurational CASSCF/RASSISO calculations have been performed on each of the four metal ions in their ligand environment, suitably disconnected from the rest of the chain (details of the calculations can be found in Ref. 7 and the accompanying Supplementary Information). Most important for us is that the $\mathrm{Dy}^{3+}$ center was found to have a ground Kramers doublet, separated by $141 \mathrm{~cm}^{-1}$ from the second doublet, and characterized by complete uniaxial anisotropy:

$$
\mathrm{Dy}^{3+}: \quad g_{z}=19.6, \quad g_{x}=0, \quad g_{y}=0 .
$$

(Actually $g_{x}$ and $g_{y}$ were calculated about 0.03 , which is small enough to be ignored.) The value of $g_{z}=19.6$ shows that this doublet is only slightly perturbed from the $\left|M_{J}= \pm 15 / 2\right\rangle$ doublet of the ${ }^{6} \mathrm{H}_{15 / 2}$ level, the latter having $g_{z}=20$. Together with the fact that the energy gap to the second Kramers doublet is about ten times larger than the exchange interaction (as we will find later), these results indicate that the $\mathrm{Dy}^{3+}$ ion will behave as an Ising spin, as described in Section IIB. As 
a side-note we may add that the total splitting of the ${ }^{6} \mathrm{H}_{15 / 2}$ level was calculated to be $560 \mathrm{~cm}^{-1}$, which is indeed of the order of room-temperature $k T$ (see Section IIE.

Both $\mathrm{Cu}^{2+}\left(d^{9}\right)$ and $\mathrm{Mo}^{5+}\left(d^{1}\right)$ have a spin $=1 / 2$, orbitally nondegenerate ground state, well separated (> $15000 \mathrm{~cm}^{-1}$ ) from higher states. The two $\mathrm{Cu}^{2+}$ ions in the unit cell reside in almost identical environments ${ }^{7}$ and have therefore virtually the same properties. The calculated $g$ factors are tetragonal: $g_{\|}=2.33, g_{\perp}=2.07$ for $\mathrm{Cu}^{2+}$ and $g_{\|}=2.00, g_{\perp}=1.95$ for $\mathrm{Mo}^{5+}$. To avoid unnecessary complications we will regard these ions as isotropic spins with root-mean-square $g$ factors

$$
\begin{array}{ll}
\mathrm{Cu}^{2+}: & g_{\mathrm{Cu}}=2.16, \\
\mathrm{Mo}^{5+}: & g_{\mathrm{Mo}}=1.97 .
\end{array}
$$

This approximation will not have important consequences for the magnetic properties, which are largely dominated by the high $\mathrm{Dy}^{3+}$ moment anyway.

We introduce exchange interaction between metal ions directly connected by ligand bridges. $\mathrm{Dy}^{3+}$ interacts with its three neighbors via the Ising Hamiltonian Eq. (14). Interaction between the isotropic spins is given by the Heisenberg Hamiltonian $-J_{i j} \mathbf{S}_{i} \cdot \mathbf{S}_{j}$. Fig. 5 shows the exchange configuration, with single bonds representing Ising interaction and double bonds representing Heisenberg interaction. Note that we have approximated the $\mathrm{Dy}-\mathrm{Cu}_{1}$ and $\mathrm{Dy}-\mathrm{Cu}_{2}$ coupling strengths to be equal $\left(J_{1}\right)$, following the approximate local symmetry of the Dy-Cu pairs. ${ }^{7}$

It is now possible to see that the $[\mathrm{DyCuMoCu}]_{\infty}$ polymeric chain is indeed an experimental realization of an Ising-Heisenberg chain where the $[\mathrm{CuMoCu}]$ trimeric Heisenberg units decorate the Dy-Dy bonds and the $\mathrm{Dy}^{3+}$ Ising spins separate the $[\mathrm{CuMoCu}]$ Heisenberg trimers from each other. The total Hamiltonian in a magnetic field $\mathbf{B}$ is then given by Eq. (1) and

$$
\begin{aligned}
\hat{h}_{i}\left(s_{i}^{z}, s_{i+1}^{z}\right)= & -J_{1} s_{i}^{z} S_{i \mathrm{Cu}_{1}}^{z}-J_{2} s_{i}^{z} S_{i \mathrm{Mo}}^{z}-J_{3} \mathbf{S}_{i \mathrm{Cu}_{1}} \cdot \mathbf{S}_{i \mathrm{Mo}} \\
& -J_{4} \mathbf{S}_{i \mathrm{Mo}} \cdot \mathbf{S}_{i \mathrm{Cu}_{2}}-J_{1} S_{i \mathrm{Cu}_{2}}^{z} s_{i+1}^{z} \\
& -\mu_{\mathrm{B}} g_{\mathrm{Dy}} s_{i}^{z} B_{z}-\mu_{\mathrm{B}}\left(g_{\mathrm{Cu}} \mathbf{S}_{i \mathrm{Cu}_{1}}+g_{\mathrm{Mo}} \mathbf{S}_{i \mathrm{Mo}}\right. \\
& \left.+g_{\mathrm{Cu}} \mathbf{S}_{i \mathrm{Cu}_{2}}\right) \cdot \mathbf{B}
\end{aligned}
$$

where $s_{i}^{z}$ is shorthand for $s_{i \mathrm{Dy}}^{z}$ and $g_{\mathrm{Dy}}$ is the $g_{z}$ factor of $\mathrm{Dy}^{3+}$ [Eq. (27)]. The $z$ axis is the anisotropy axis of the $\mathrm{Dy}^{3+}$ center. We have not specified its direction with respect to the chain axis but this is not important here because there are no other axes in the problem (the Dy ${ }^{3+}$ anisotropy axes are parallel by translational symmetry and we have assumed $\mathrm{Cu}^{2+}$ and $\mathrm{Mo}^{5+}$ isotropic). All the spins in Eq. 28) are spins of $1 / 2$.

The Hamiltonian exhibits some symmetry. It is rotationally invariant around $z$, if $\mathbf{B}$ is rotated simultaneously. We may therefore restrict $\mathbf{B}$ to lie in a plane through $z$, say the $x z$ plane. This simplifies calculation

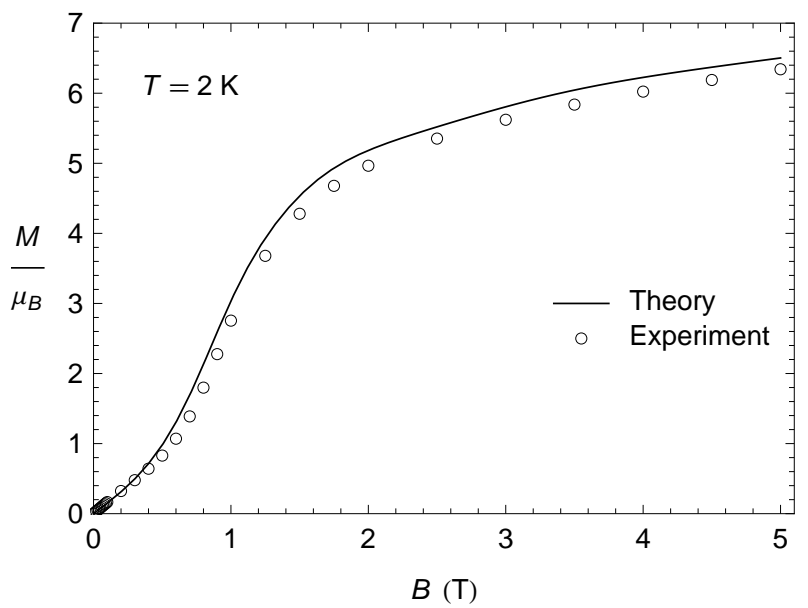

FIG. 6. Powder magnetization of $[\mathrm{DyCuMoCu}]_{\infty}$.

of the powder magnetization Eq. 22): one has to integrate only over $\theta$. When $\mathbf{B}$ is directed along the $z$ axis, the $z$ component of the total spin in each decorating unit is conserved:

$$
S_{i}^{z}=S_{i \mathrm{Cu}_{1}}^{z}+S_{i \mathrm{Mo}}^{z}+S_{i \mathrm{Cu}_{2}}^{z}, \quad\left[S_{i}^{z}, \hat{H}(\mathbf{B} \| \hat{\mathbf{z}})\right]=0 .
$$

We also note that in this case the Zeeman Hamiltonian commutes almost with $\hat{H}$. It would commute exactly when $g_{\mathrm{Cu}}=g_{\mathrm{Mo}}$, for then the last term in Eq. (28) reduces to $-\mu_{\mathrm{B}} g_{\mathrm{Cu}} S_{i}^{z} B_{z}$.

We let the length of the chain go to infinity: $n \rightarrow \infty$. To solve for the thermodynamic properties we are only required to find the eigenvalues $\varepsilon_{k}\left(s, s^{\prime}\right)$ of Eq. (28) (See Section II A), with $k=1 \ldots 8$, corresponding to the $2^{3}$ possible states of the $[\mathrm{CuMoCu}]$ spin unit. This is done by 4 numerical $8 \times 8$ matrix diagonalizations, one for each $\left(s, s^{\prime}\right)$ pair.

We can now compare the theory with experiment. Powder magnetization (at $2 \mathrm{~K}$ ) and susceptibility data have been recorded ${ }^{7}$ We recall that we have to correct the theoretical curves before comparing with experiment according to Eq. (26). The corrections are provided by the ab initio calculations. ${ }^{7} \mu_{\text {Dy }}^{\prime}$ turns out to be $0.03 \mu_{\mathrm{B}} / \mathrm{T}$; the accompanying correction in Eq. 26b is never more than $2.5 \%$ of $M$. We ignore this correction. We do however correct $\chi$ as in Eq. (26a).

Closest agreement with experiment was found for the following values of the exchange constants (plots in Figs. 6 and 7):

$$
\begin{aligned}
& J_{1}=15.3 \mathrm{~cm}^{-1}, J_{2}=-8.0 \mathrm{~cm}^{-1}, J_{3}=-8.3 \mathrm{~cm}^{-1}, \\
& J_{4}=11.8 \mathrm{~cm}^{-1} .
\end{aligned}
$$

These were obtained by a least-squares fit of $\chi$ followed by a small manual adjustment to improve the fit of $M$ while not distorting that of $\chi$ appreciably. (The leastsquares fit of $\chi$ gave $J_{1}=15.7 \mathrm{~cm}^{-1}, J_{2}=-8.3 \mathrm{~cm}^{-1}$, $J_{3}=-6.3 \mathrm{~cm}^{-1}, J_{4}=11.8 \mathrm{~cm}^{-1}$.) There are, as far as 


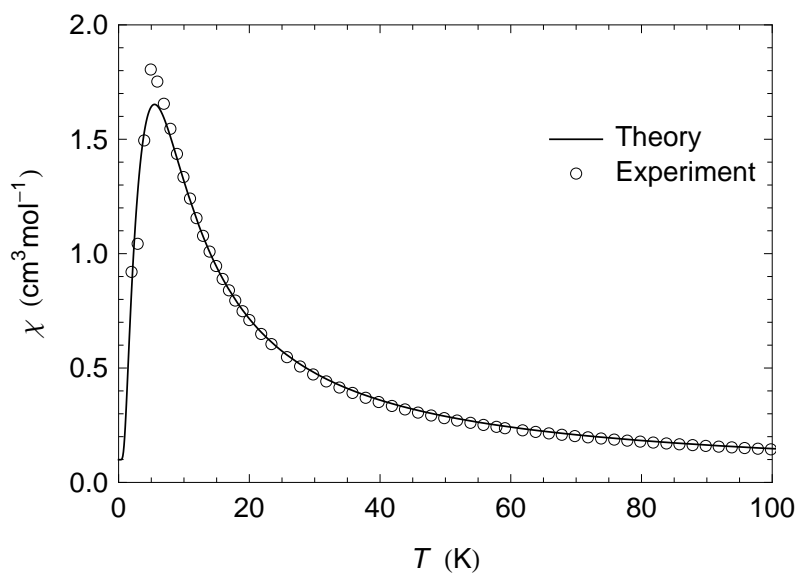

FIG. 7. Powder magnetic susceptibility of $[\mathrm{DyCuMoCu}]_{\infty}$. The theoretical curve contains the correction for the contribution of excited Kramers doublets.

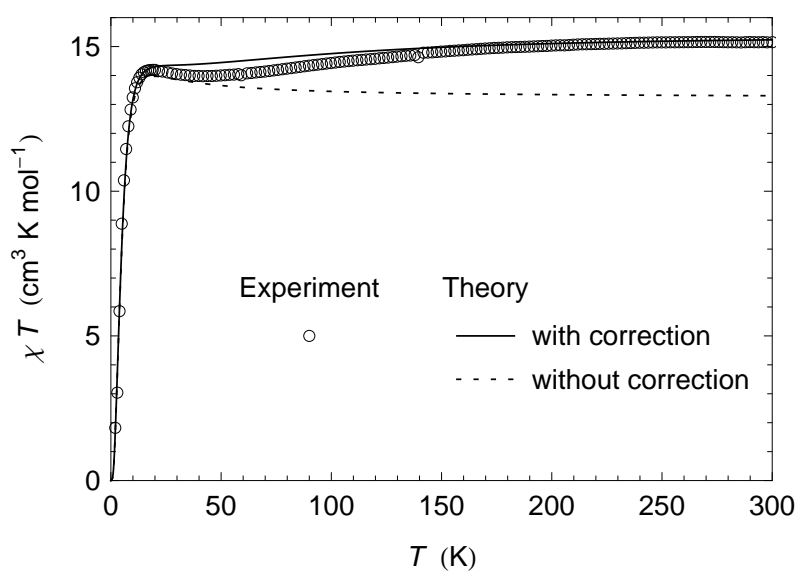

FIG. 8. Powder magnetic susceptibility of $[\mathrm{DyCuMoCu}]_{\infty}$. The correction refers to the contribution of excited Kramers doublets, Eq. 26a.

we know, no data in the literature with which to compare the values in Eq. (30). However, an experimental study is available of a $\left(\mathrm{Dy}^{3+}, \mathrm{Cu}^{2+}\right)$ dinuclear complex in which the bridging ligand is the same as in this chain.26 The authors found a ferromagnetic interaction. A superficial analysis of the susceptibility curve in that paper, using the Ising Hamiltonian we use in this paper, yields $J_{1}=$ $15 \pm 5 \mathrm{~cm}^{-1}$. The other values in (30) are difficult to assess. For a discussion of these values and their relation with the molecular structure as well as some evidence from DFT calculations, we refer to Ref. 7. Certainly, no confidence should be attached to the numbers in decimal places in (30).

The effect of the excited Kramers doublets of $\mathrm{Dy}^{3+}$ is most clearly seen in the $\chi T$ curve (Fig. 8). The curve shows a steady increase above $50 \mathrm{~K}$ which is not predicted by our decorated Ising model, but is indeed due to the thermal population of the Kramers doublets that originate from the ${ }^{6} \mathrm{H}_{15 / 2}$ level. We can obtain the ex- pected high-temperature limit of $\chi T$ by considering the metal ions as independent spins. The susceptibility components $\chi_{\alpha \alpha}$ of an angular momentum multiplet $J$ with principal $g$-factors $g_{\alpha}(\alpha=x, y, z)$ are given by 27

$$
\chi_{\alpha \alpha}=\frac{N_{A} \mu_{\mathrm{B}}^{2}}{3 k T} g_{\alpha}^{2} J(J+1) .
$$

Summing over Dy ${ }^{3+}\left({ }^{6} \mathrm{H}_{15 / 2}, g_{15 / 2}=4 / 3\right), \mathrm{Cu}_{1}, \mathrm{Mo}$, and $\mathrm{Cu}_{2}$ (all are isotropic) gives

$$
\begin{aligned}
\chi T & =\frac{1}{3} \sum_{\alpha} \chi_{\alpha \alpha} T \\
& =\frac{N_{A} \mu_{\mathrm{B}}^{2}}{3 k}\left(g_{15 / 2}^{2} \frac{15}{2} \frac{17}{2}+\left(g_{\mathrm{Cu}}^{2}+g_{\mathrm{Mo}}^{2}+g_{\mathrm{Cu}}^{2}\right) \frac{13}{2} \frac{3}{2}\right) \\
& =15.4 \frac{\mathrm{cm}^{3} \mathrm{~K}}{\mathrm{~mol}} .
\end{aligned}
$$

A similar calculation, only including the lowest Kramers doublet of $\mathrm{Dy}^{3+}$, with $g$-factors as in Eq. (27), gives $13.2 \mathrm{~cm}^{3} \mathrm{~K} \mathrm{~mol}^{-1}$. The correction supplied by the $a b$ initio calculations to account for this difference, is seen to cover nicely the high-temperature part of the experimental curve.

One notices that $\chi T$ shows a slight depression around $40 \mathrm{~K}$ which is not entirely reproduced by the theory. This might indicate a failure of the simple approximation we used to include the excited Kramers doublets. Eq. (26a) is certainly correct at very high temperatures, when the exchange interactions are irrelevant, and at very low temperatures, when the excited Kramers doublets are not occupied. If these two regions do not overlap, however, there is a temperature window between, in which excited doublets start to get occupied while exchange interaction is not quite negligible yet. In that case, the exchange interaction of the occupied excited doublet(s) with other ions should be taken into account. Such an interaction of antiferromagnetic type could possibly depress $\chi T$ as observed.

We shall now describe some features of the spectrum of the chain, paying attention to the properties described in Section IIC] Consider the chain without magnetic field. The exchange parameters in Eq. (30) predict a ground state that has an AF Ising spin configuration. This is in accordance with the susceptibility measurements, which show that $\chi T \rightarrow 0$ as $T \rightarrow 0$, requiring a nonmagnetic ground state (Fig. 8). The ground state is indeed nonmagnetic because $\left|\varepsilon_{1}(\uparrow \downarrow)\right\rangle$ is the time-reversed state of $\left|\varepsilon_{1}(\downarrow \uparrow)\right\rangle$. Let $M_{S}$ denote an eigenvalue of $S^{z}$ [Eq. $\left.(29)\right]$ : $M_{S} \in\{-3 / 2,-1 / 2,1 / 2,3 / 2\} . \quad S_{i}^{z}$ is conserved so $M_{S}$ may be used to label the eigenstates $\left|\varepsilon_{k}\left(s, s^{\prime}\right)\right\rangle$ (we may leave out the index $i$ because all units of the chain are identical). For the ground state, we find $M_{S}=-1 / 2$ in $\left|\varepsilon_{1}(\uparrow \downarrow)\right\rangle$ and $M_{S}=1 / 2$ in $\left|\varepsilon_{1}(\downarrow \uparrow)\right\rangle$.

Since the ground state is AF, we might expect that in a magnetic field a crossover will occur to an $\mathrm{F}$ ground state. This is indeed what happens. The convex increase of $M$ in Fig. 6 points to a flip of the $\mathrm{Dy}^{3+}$ spins to a 


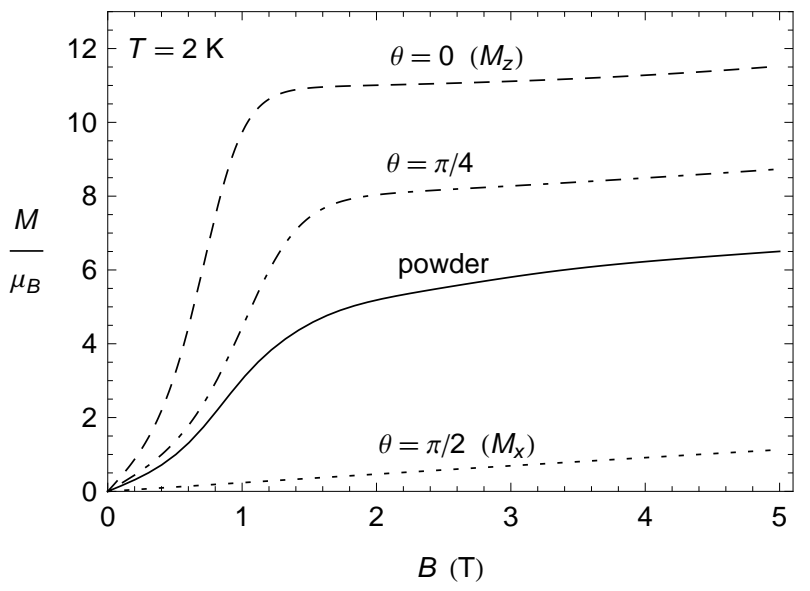

FIG. 9. Theoretical magnetization of $[\mathrm{DyCuMoCu}]_{\infty}$. The powder magnetization (See also Fig. 6) is compared with the projections of $\mathbf{M}$ on the field direction [Eq. (21)], for three different directions of the field; $\theta$ is the angle between $\mathbf{B}$ and the $z$ axis.

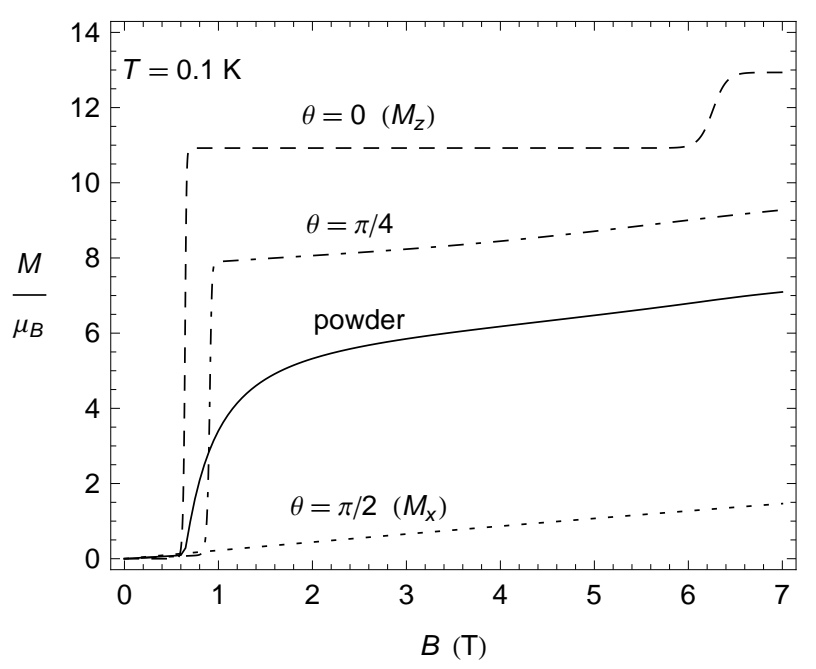

FIG. 10. Theoretical magnetization of $[\mathrm{DyCuMoCu}]_{\infty}$. Same as Fig. 9 but at lower temperature and to higher field.

parallel configuration. This is inferred from the value of the magnetization, which approaches $6 \mu_{\mathrm{B}}$ at $5 \mathrm{~T}$. The $[\mathrm{CuMoCu}]$ unit alone can only contribute a maximum of $(2.16+1.97+2.16) / 2=3.15 \mu_{\mathrm{B}}$. The strong increase must come from the contribution of the large $\mathrm{Dy}^{3+}$ moments.

The behavior of magnetization along certain directions of applied field is shown in Fig. 9. The $\mathrm{AF} \rightarrow \mathrm{F}$ transition is most clearly seen when the field is applied along $z$ $(\theta=0)$; the transition occurs below $1 \mathrm{~T}$. After $1 \mathrm{~T}, M_{z}$ reaches an approximately constant plateau at $\approx 11 \mu_{\mathrm{B}}$. The saturation value of magnetization in direction $0 \leq$ $\theta \leq \pi / 2$ is $(19.6 \cos \theta+2.16+1.97+2.16) / 2$. This gives $12.9 \mu_{\mathrm{B}}$ for $\theta=0$, which shows that $M_{z}$ has not quite reached its maximum at $5 \mathrm{~T}$ yet.

The positions of level crossings become more sharply defined on lowering the temperature (Fig. 10). Here we

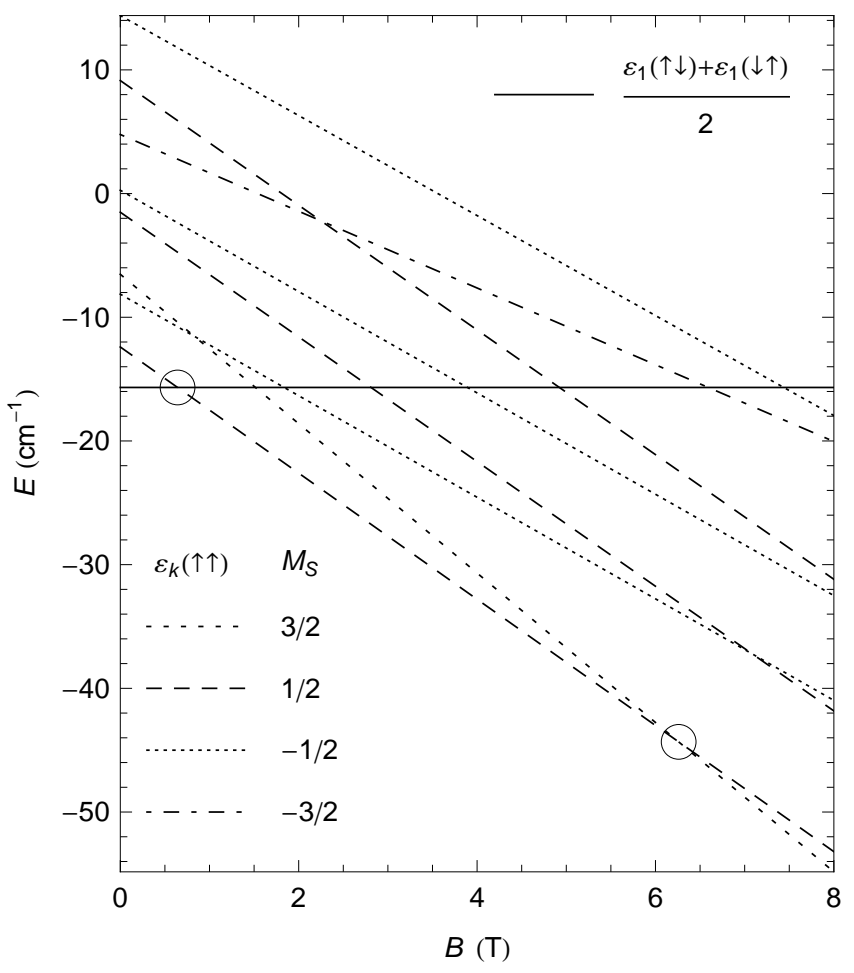

FIG. 11. Eigenvalues of $\hat{h}\left(s, s^{\prime}\right)$ [Eq. 28)] in a magnetic field parallel with the $z$ axis $(\theta=0)$. Circles indicate ground state level crossings. The ground state of the chain is AF in zero field (left), switches to $\mathrm{F}$ at $0.64 \mathrm{~T}$, and undergoes an internal level crossing at $6.3 \mathrm{~T}$, marked by a change of the internal quantum number $M_{S}$ from $1 / 2$ to $3 / 2$. Both crossings can be seen in the $\theta=0$ magnetization curve in Fig. 10 Note that the energy curves appear as straight lines, although, with the exception of $M_{S}= \pm 3 / 2$, they are not exactly straight, because the Zeeman Hamiltonian does not completely commute with the total Hamiltonian. All $\varepsilon_{k}(\uparrow \uparrow$ ) decrease with increasing field strength because the large magnetic moment of $\mathrm{Dy}^{3+}$ dominates the smaller magnetic moments of the decorating unit.

also see that $M_{z}$ undergoes a second transition at $6.3 \mathrm{~T}$, after which it reaches saturation. This transition is connected with a level crossing in the $[\mathrm{CuMoCu}]$ unit (see Section II C) from $M_{S}=1 / 2$ to $M_{S}=3 / 2$, as opposed to the first transition, at $0.64 \mathrm{~T}$, which is of the Ising type, described by Eq. 20). The latter is the analogue of the transition in the AF simple Ising chain (Fig. 3), while the "internal" transition has no such analogue but is unique to the decorated Ising chain. The relevant energy level diagram is shown in Fig. 11. Note that, for fields not parallel to $z$ (for example, $\theta=\pi / 4$ in Fig. 10), $M_{S}$ is not a quantum number and the internal level crossing turns into an avoided crossing. This does not apply for the Ising level crossing because the Ising spins are always conserved. Only when the field is applied perpendicular to $z(\theta=\pi / 2$ in Fig. 10$)$ does the $\mathrm{AF} \rightarrow \mathrm{F}$ transition not occur because the $\mathrm{Dy}^{3+}$ spins do not interact with perpendicular fields. 


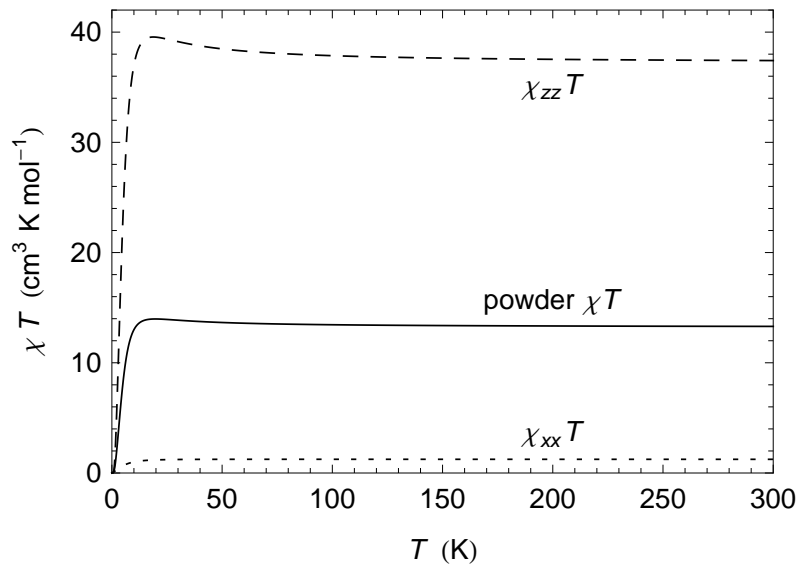

FIG. 12. Theoretical susceptibility of $[\mathrm{DyCuMoCu}]_{\infty}$, without correction for contribution of excited Kramers doublets. The powder $\chi T$ (see also Fig. 8) is compared with the Cartesian components of $\chi T . z$ is the direction of the anisotropy axis of $\mathrm{Dy}^{3+}, x$ is any direction perpendicular to $z$. $\chi=$ $\left(\chi_{z z}+2 \chi_{x x}\right) / 3$.

The low-temperature limit of the powder magnetization in Fig. 10 may be compared with that of the simple Ising chain in Fig. 4. The resemblance is clear; the decorated chain is different in the small linear increase of $M$ before the transition, and the more linear approach to saturation, which lies at $(19.6 / 2+2.16+1.97+2.16) / 2=$ $8.0 \mu_{\mathrm{B}}$. Both are due to TIP interaction in the [CuMoCu] unit, the effect of which is most clearly seen in the $M_{x}$ curve in Fig. 10 .

To conclude this section we remark that the mentioned similarity with the magnetization of the simple Ising chain is a consequence of the very high magnetic moment of the $\mathrm{Dy}^{3+}$ spins in comparison with the $[\mathrm{CuMoCu}]$ unit. The dominance of $\mathrm{Dy}^{3+}$ is most dramatically shown in the components of $\chi T$ (Fig. 12). An application of Eq. (31) shows that the high-temperature limit of $\chi_{x x} T$ is $\frac{N_{A} \mu_{\mathrm{B}}^{2}}{3 k}\left(2.16^{2}+1.97^{2}+2.16^{2}\right) \frac{1}{2} \frac{3}{2}=1.24 \mathrm{~cm}^{3} \mathrm{~K} \mathrm{~mol}^{-1}$, while that of $\chi_{z z} T$ is $\frac{N_{A} \mu_{\mathrm{B}}^{2}}{3 k}\left(19.6^{2}+2.16^{2}+1.97^{2}+2.16^{2}\right) \frac{1}{2} \frac{3}{2}=$ $37.2 \mathrm{~cm}^{3} \mathrm{~K} \mathrm{~mol}^{-1}$.

\section{IV. $\mathrm{Dy}_{4} \mathrm{Cr}_{4}$ COMPLEX AS DECORATED ISING RING}

As a second example we describe in this section the application of the decorated Ising model to a ring-shaped $\mathrm{Dy}_{4} \mathrm{Cr}_{4}$ molecule ${ }^{8} \mathrm{Dy}_{4} \mathrm{Cr}_{4}$ consists of alternating Dy ${ }^{3+}$ and $\mathrm{Cr}^{3+}$ ions forming a closed ring. The four Dy ${ }^{3+}$ ions lie in a plane. The $\mathrm{Cr}^{3+}$ ions are positioned alternatingly above and below this plane, "decorating" the DyDy bonds. The molecule has $D_{2 d}$ symmetry, the Dy ${ }^{3+}$ ions lying on $C_{2}$ axes and the $\mathrm{Cr}^{3+}$ ions lying on the mirror planes. We choose a molecular reference frame $X Y Z$ so that $Z$ coincides with the $S_{4}$ axis and $X$ and $Y$ coincide with the two $C_{2}$ axes of $D_{2 d}$ (Fig. 13).

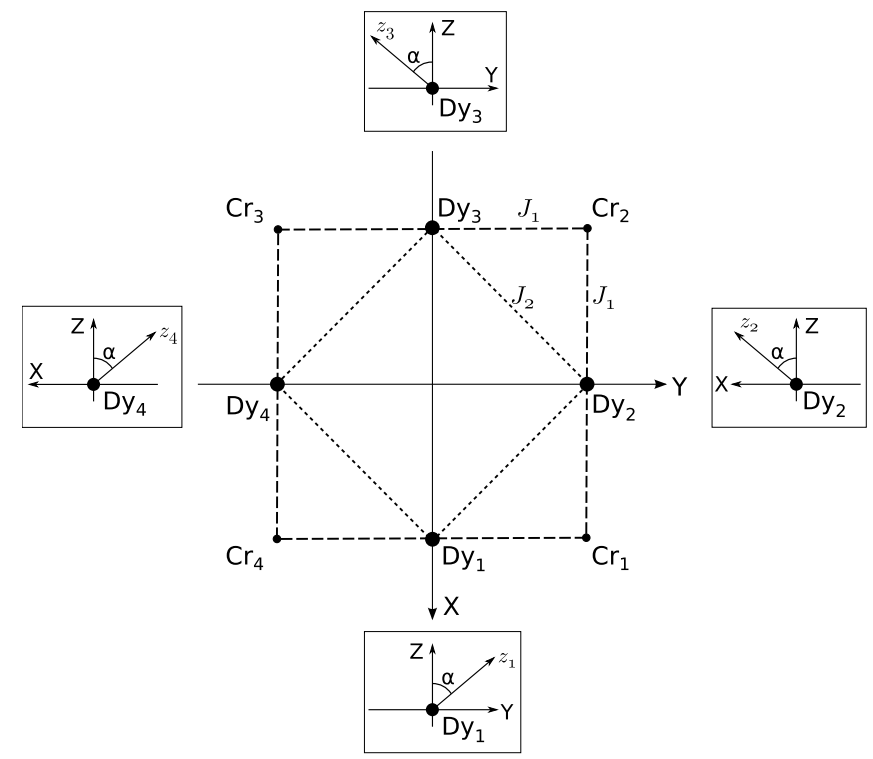

FIG. 13. Schematic representation of the $\mathrm{Dy}_{4} \mathrm{Cr}_{4}$ molecule indicating numbering of atoms and exchange coupling constants. The boxes show the orientation of the local anisotropy axes on Dy sites, when viewed from the poles of the $X$ and $Y$ axes. The $Z$ axis points out of the center of the scheme.

$A b$ initio calculations have been performed in the same way as for the $[\mathrm{DyCuMoCu}]$ chain. ${ }^{8}$ From these, we take again the $g$-factors of the ground doublet of $\mathrm{Dy}^{3+}$ and of the isotropic ground state spin multiplet of $\mathrm{Cr}^{3+}\left(d^{3}\right.$, $S=3 / 2)$ :

$$
\begin{array}{ll}
\mathrm{Dy}^{3+}: & g_{z}=19.66, \quad g_{x}=0, \quad g_{y}=0, \\
\mathrm{Cr}^{3+}: & g_{\mathrm{Cr}}=1.97 .
\end{array}
$$

The Dy ${ }^{3+}$ Kramers doublet is again very close to the $\left|M_{J}= \pm 15 / 2\right\rangle$ state, permitting the use of the Ising model. However, the same calculation predicted the second Kramers doublet at $30 \mathrm{~cm}^{-1}$, not very high compared with exchange interaction, which we found in the previous section $\approx 10 \mathrm{~cm}^{-1}$. This should be seen as a warning that our treatment of the excited Kramers doublets as "innocent" may not be entirely correct here, and thus may lead to discrepancies with experiment. In this respect we must also note that, for such small excitation energies, the results of the $a b$ initio calculations are not always conclusive on the nature of the ground state Kramers doublet. In the present case, for instance, another set of calculations produced a ground state Kramers doublet on $\mathrm{Dy}^{3+}$ that is not uniaxial as in Eq. (32), having relatively large transversal $g$-factors $\frac{8}{8}$ The decorated Ising model would be unusable in this case. We find however that, assuming the axial g-factors in Eq. (32), $\mathrm{Dy}_{4} \mathrm{Cr}_{4}$ is an interesting example of a decorated Ising ring, for which qualitative agreement with experimental magnetic properties can be obtained.

Direct ligand bridges connect each $\mathrm{Dy}^{3+}$ with two neighboring $\mathrm{Cr}^{3+}$ ions and two neighboring $\mathrm{Dy}^{3+}$ ions. 
Exchange interaction between these pairs is introduced [Eqs (13) and (14)]. The Hamiltonian is then given by Eq. (1): $\hat{H}=\hat{h}_{1}+\hat{h}_{2}+\hat{h}_{3}+\hat{h}_{4}$, and

$$
\begin{aligned}
\hat{h}_{i}\left(s_{i}^{z_{i}}, s_{i+1}^{z_{i+1}}\right)= & -J_{1}\left(s_{i}^{z_{i}} S_{i}^{z_{i}}+s_{i+1}^{z_{i+1}} S_{i}^{z_{i+1}}\right)-J_{2} s_{i}^{z_{i}} s_{i+1}^{z_{i+1}} \\
& -\mu_{\mathrm{B}}\left(g_{\mathrm{Dy}} s_{i}^{z_{i}} B^{z_{i}}+g_{\mathrm{Cr}} \mathbf{S}_{i} \cdot \mathbf{B}\right),
\end{aligned}
$$

where $s_{i}^{z_{i}}$ denotes the Ising spin- $1 / 2$ variable on $\mathrm{Dy}_{i}$ and $S_{i}^{z_{i}}$ denotes the projection of the spin of $\mathrm{Cr}_{i}$ on the magnetic anisotropy axis of $\mathrm{Dy}_{i}$ (for numbering, see Fig 13). Similarly, $B^{z_{i}}$ is the projection of the magnetic field on the anisotropy axis of $\mathrm{Dy}_{i} . g_{\mathrm{Dy}}$ is the $g_{z}$ factor of $\mathrm{Dy}^{3+}$ [Eq. [32] ].

An interesting difference with the $[\mathrm{DyCuMoCu}]$ chain is that here, in $\mathrm{Dy}_{4} \mathrm{Cr}_{4}$, the four anisotropy axes $z_{i}$ are not, in general, parallel, a result of point symmetry instead of translational symmetry. The orientation of the local anisotropy axis on $\mathrm{Dy}^{3+}$, being one of the $g$-tensor main axes, is restricted by the local $C_{2}$ symmetry to be either parallel with, or orthogonal to the local $C_{2}$ axis. The first possibility can be excluded on the basis of the experiment; with the $z_{i}$ pointing radially outwards at each $\mathrm{Dy}_{i}$, the ground state of the whole molecule is necessarily nonmagnetic, because the local moments add up to zero, independent of whether the ground state is $\mathrm{F}$ or AF with respect to the Ising spins. The experimental susceptibility measurement however indicates a magnetic ground state (nonzero intercept on the vertical axis in Fig. 14). We must therefore choose the second case and let the anisotropy axis on each Dy be orthogonal to the local $C_{2}$ axis and make an angle of $\alpha$ with the molecular $Z$ axis (See Fig. 13). By applying the symmetry elements of $D_{2 d}$ to one of these anisotropy axes, one obtains the other three. When $\alpha=0$ the four axes are parallel and point in the same direction as $Z$. We note that the $a b$ initio calculations yielded $\alpha=37^{\circ}$. We will need some flexibility in our model however, so we leave $\alpha$ as a parameter that will be determined from comparison with experiment.

In terms of the molecular coordinate system, the projections on the local anisotropy axes are a function of $\alpha$ :

$$
\begin{aligned}
S_{i}^{z_{1}} & =\cos (\alpha) S_{i}^{Z}+\sin (\alpha) S_{i}^{Y} \\
S_{i}^{z_{2}} & =\cos (\alpha) S_{i}^{Z}+\sin (\alpha) S_{i}^{X} \\
S_{i}^{z_{3}} & =\cos (\alpha) S_{i}^{Z}-\sin (\alpha) S_{i}^{Y} \\
S_{i}^{z_{4}} & =\cos (\alpha) S_{i}^{Z}-\sin (\alpha) S_{i}^{X} .
\end{aligned}
$$

The same relations hold for the magnetic field, after replacing $S_{i}$ by $B$.

The fact that only exchange interactions of Ising type appear in Eq. (33) makes it possible to find analytical solutions of the eigenvalues and the partition function. From Eqs. (33) and (34) we see that the part of $\hat{h}_{i}$ that involves $S_{i}$ is a projection of $\mathbf{S}_{i}$ on the vector

$$
-J_{1}\left(s_{i} \hat{\mathbf{z}}_{i}+s_{i+1} \hat{\mathbf{z}}_{i+1}\right)-g_{\mathrm{Cr}} \mu_{\mathrm{B}} \mathbf{B},
$$

where $\hat{\mathbf{z}}_{i}$ is the unit vector along the anisotropy axis of $\mathrm{Dy}_{i}$ (the superscripts $z_{i}$ on $s_{i}$ are left out from now on). The vector (35) defines the quantization axis of $\mathbf{S}_{i}$, which depends on the states on the neighboring $\mathrm{Dy}^{3+}$ sites $\left(s_{i}, s_{i+1}\right)$. The stronger the coupling $\left(J_{1}\right)$ with Dy, the stronger will be the deviation of the quantization axis from the direction of $\mathbf{B}$. The eigenvalues of $\hat{h}_{i}$ are then

$$
\varepsilon_{i M_{S}}\left(s_{i}, s_{i+1}\right)=b_{i} M_{S}-J_{2} s_{i} s_{i+1}-\mu_{\mathrm{B}} g_{\mathrm{Dy}} s_{i} B^{z_{i}},
$$

where $M_{S}=-S, \ldots, S$, and

$$
b_{i}=\sqrt{\frac{J_{1}^{2}}{2}\left(1+4 s_{i} s_{i+1} \cos ^{2} \alpha\right)+2 J_{1} \mu_{\mathrm{B}} g_{\mathrm{Cr}}\left(s_{i} B^{z_{i}}+s_{i+1} B^{z_{i+1}}\right)+\mu_{\mathrm{B}}^{2} g_{\mathrm{Cr}}^{2} B^{2}}
$$

is the length of the vector in Eq. (35). Some remarks should be made on the solutions. Eqs. (37) and (34) (replace $S_{i}$ by $B$ ) show that the spectrum in Eq. (36) is not the same for every unit $i$, as it was in the [DyCuMoCu] chain, unless $\mathbf{B}$ is applied along the $Z$ axis. This means that also the transfer matrices $T_{i}$ will be different and that we have to use Eq. (7) instead of Eq. (8) for the partition function. A second remark concerns the quantum number $M_{S}$. The lowest energy in Eq. (36) is always given by $M_{S}=-S$, but note that the axis to which this quantization refers is not invariant; in particular, it changes with strength and direction of applied field, so that $M_{S}$ does not represent a real conserved quantity that could be responsible for level crossings of the "internal" type. Such crossings do not occur in $\mathrm{Dy}_{4} \mathrm{Cr}_{4}$.
We conclude the solution by finding the partition function $\mathcal{Z}$. Substituting Eq. (36) in Eq. (4) we find

$$
\begin{aligned}
\Psi_{i}\left(s_{i}, s_{i+1}\right)= & \sum_{M_{S}=-S}^{S} e^{-\beta \varepsilon_{i M_{S}}} \\
= & \frac{\sinh \left[\beta b_{i}(2 S+1) / 2\right]}{\sinh \left[\beta b_{i} / 2\right]} \\
& \times \exp \left[\beta\left(J_{2} s_{i} s_{i+1}+\mu_{\mathrm{B}} g_{\text {Dy }} s_{i} B^{z_{i}}\right)\right]
\end{aligned}
$$

With $T_{i}$ as defined in Eq. (6), we obtain the partition function

$$
\mathcal{Z}=\operatorname{Tr}\left(T_{1} T_{2} T_{3} T_{4}\right)
$$

Let us now compare the theoretical results with experiment. A great amount of information on the values of the 
parameters $\alpha, J_{1}$ and $J_{2}$ can be obtained by inspection of the powder $\chi T$ curve (Fig. 14). The nonzero intercept $\left.\chi T\right|_{T \rightarrow 0}=34.6 \mathrm{~cm}^{3} \mathrm{~K} \mathrm{~mol}^{-1}$ indicates a magnetic ground state. ${ }^{27}$ Now from the general theory we know that the ground state is either $\mathrm{F}(\uparrow \uparrow \uparrow \uparrow)$ or $\mathrm{AF}(\uparrow \downarrow \uparrow \downarrow)$ with respect to the $\mathrm{Dy}^{3+}$ spins, $\stackrel{28}{,} \mathrm{AF}$ is nonmagnetic so we decide that the ground state must be F.

Incidentally, we can precisely delineate the regions in parameter space where the ground state is $\mathrm{F}$ or AF:

$$
\begin{gathered}
\mathrm{F}(\uparrow \uparrow \uparrow \uparrow): J_{2}>-\sqrt{2} S\left(\sqrt{1+\cos ^{2} \alpha}-\sin \alpha\right)\left|J_{1}\right|, \\
\mathrm{AF}(\uparrow \downarrow \uparrow \downarrow): J_{2}<-\sqrt{2} S\left(\sqrt{1+\cos ^{2} \alpha}-\sin \alpha\right)\left|J_{1}\right| .
\end{gathered}
$$

A second piece of information comes from the increase of $\chi T$ with increasing temperature. This is partly but not completely due to the occupation of excited Kramers doublets, as one can show by subtracting the contribution of the latter, obtained from the ab initio calculations (not shown here). There must still be an antiferromagnetic interaction to explain the increase. Since the $\mathrm{Dy}^{3+}$ are already known to be ferromagnetically aligned, the only possibility is that the $\mathrm{Cr}^{3+}$ spins couple antiferromagnetically with $\mathrm{Dy}^{3+}$, or $J_{1}<0$.

With this information, we can determine the angle $\alpha$. At $0 \mathrm{~K}, \chi T$ is determined by the magnetic moment in the ground state only ${ }^{27 / 29]}$ In the $\mathrm{F}$ ( $\left.\uparrow \uparrow \uparrow \uparrow\right)$ state, $\left.\chi_{X X} T\right|_{T \rightarrow 0}=\left.\chi_{Y Y} T\right|_{T \rightarrow 0}=0$ by symmetry and

$\left.\chi_{Z Z} T\right|_{T \rightarrow 0}=\frac{N_{A} \mu_{\mathrm{B}}^{2}}{k}\left|\left\langle F\left|g_{\text {Dy }} \cos \alpha \sum_{i=1}^{4} s_{i}+g_{\mathrm{Cr}} \sum_{i=1}^{4} S_{i}^{Z}\right| F\right\rangle\right|^{2}$,

so $\left.\chi T\right|_{T \rightarrow 0}=\left.\frac{1}{3} \chi_{Z Z} T\right|_{T \rightarrow 0}$. With the help of Eqs. (34)(37) and the fact that, in the ground state, $M_{S}=-S$ in Eq. (36), we can evaluate Eq. (39) to find

$$
\begin{aligned}
\left.\chi T\right|_{T \rightarrow 0}= & \frac{N_{\mathrm{A}} \mu_{\mathrm{B}}^{2}}{3 k} 4 \cos ^{2} \alpha\left(g_{\text {Dy }}\right. \\
& \left.+\operatorname{sgn}\left(J_{1}\right) 2 \sqrt{2} g_{\mathrm{Cr}} S \frac{1}{\sqrt{1+\cos ^{2} \alpha}}\right)^{2} .
\end{aligned}
$$

This is a strictly decreasing function of $\alpha$ that can be used to derive $\alpha$ from the experimental value $\left.\chi T\right|_{T \rightarrow 0}=$ $34.6 \mathrm{~cm}^{3} \mathrm{~K} \mathrm{~mol}^{-1}$, and the knowledge that $\operatorname{sgn}\left(J_{1}\right)=-1$. This gives $\alpha=49^{\circ}$.

We derive values for $J_{1}, J_{2}$, and $\alpha$ by a least-squares fitting of $\chi$. As before, a correction for the contribution of excited Dy ${ }^{3+}$ Kramers doublets is provided by the $a b$ initio calculations and applied following Eq. 26). The fitting yields

$$
J_{1}=-8.9 \mathrm{~cm}^{-1}, \quad J_{2}=5.2 \mathrm{~cm}^{-1}, \quad \alpha=49.2^{\circ} .
$$

The comparison with experiment is shown in Figs. 14 and 15 Note that the magnetic properties are reported per mole $(\chi$ and $\chi T)$ or per molecule $(M)$ of $\mathrm{Dy}_{4} \mathrm{Cr}_{4}$ and
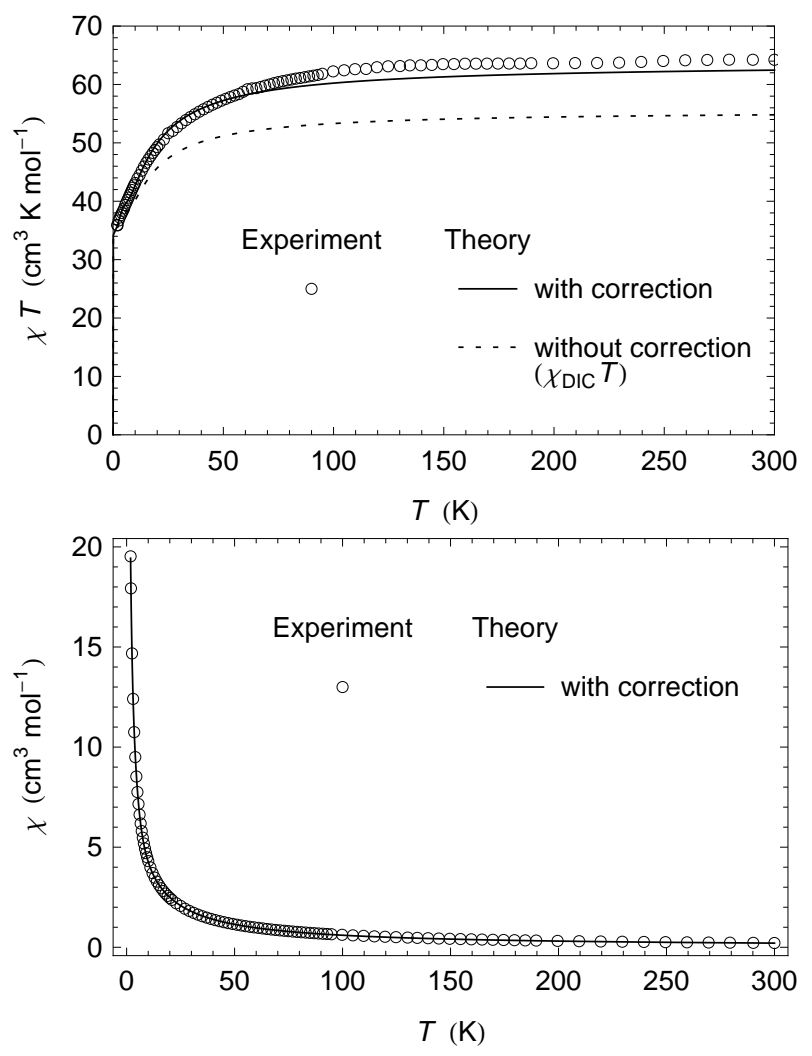

FIG. 14. Powder magnetic susceptibility of $\mathrm{Dy}_{4} \mathrm{Cr}_{4}$. The correction on the theory refers to Eq. 26.

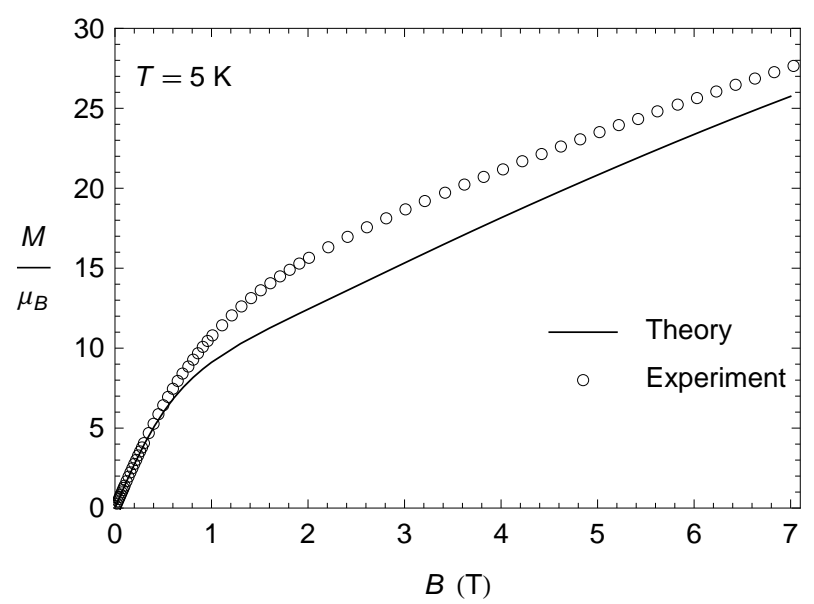

FIG. 15. Powder magnetization of $\mathrm{Dy}_{4} \mathrm{Cr}_{4}$. A linear corrections of $0.5 \mu_{\mathrm{B}} / \mathrm{T}$ has been added to the theoretical curve, according to Eq. 26b.

not per DyCr unit. Note also that $\operatorname{sgn}\left(J_{1}\right)=-1$, that $J_{2}$ satisfies Eq. (38a), and that $\alpha$ agrees with the value derived above.

The agreement of magnetization curves (Fig. 15 ) is not as good as it was for the $[\mathrm{DyCuMoCu}]_{\infty}$ chain, although the qualitative properties seem to correspond. In particular, we mention the strong linear increase of $M$ at 
higher fields $\left(\approx 2.6 \mu_{\mathrm{B}} / \mathrm{T}\right)$, which is due to the gradual orientation of the $\mathrm{Cr}^{3+}$ spins to the magnetic field [see discussion connected with Eq. (35)], and, to a smaller extent, also to the correction of $0.5 \mu_{\mathrm{B}} / \mathrm{T}$, a non-negligible linear contribution to magnetization, which is due to the low-lying excited Kramers doublets.

As was mentioned before, the discrepancies are not unexpected given a low-lying first excited Kramers doublet of $\mathrm{Dy}^{3+}$, which could undermine the assumptions underlying the decorated Ising model. Note also that we could not take the $a b$ initio value of $37^{\circ}$ for $\alpha$. Leaving $\alpha$ as a parameter can be seen as a partial compensation for the inaccuracies of the model and the $a b$ initio results.

\section{CONCLUSION}

We have shown that the decorated Ising model is a valid model for the magnetic properties of certain lanthanide-containing magnetic compounds, if the crystal field spectrum of the lanthanide ion satisfies certain properties. The most important of these is the requirement of a ground state Kramers doublet with completely uniaxial magnetic anisotropy (this statement is simplified, see Section II B for the correct details). It is a remarkable fact that precisely this property has been estab- lished by multiconfigurational $a b$ initio calculations on several $\mathrm{Dy}^{3+}$ centers that are part of polynuclear molecular magnets. Perhaps the best known example is the $\mathrm{Dy}_{3}$ triangle, where the Ising properties of $\mathrm{Dy}^{3+}$ were used to explain the nature of the ground state ${ }^{22}$

We have focused on $\mathrm{Dy}^{3+}$ as lanthanide ion because this is a much-used lanthanide in current synthetic research in molecular magnetism (witness both compounds in this paper) and because computational results showing that it meets the requirements for an Ising spin are available. However, there is no reason to assume that the findings are unique to $\mathrm{Dy}^{3+}$. We expect that other lanthanides with high momentum (e.g., $\mathrm{Er}^{3+}$ ) will exhibit the same uniaxial anisotropy in certain ligand environments and that examples of decorated Ising chains based on lanthanides other than $\mathrm{Dy}^{3+}$ will be found in the future.

\section{ACKNOWLEDGMENTS}

We thank Liviu Ungur for providing results of the $a b$ initio calculations. We thank the referee for useful suggestions and comments. W. V.d.H. acknowledges financial support from the Research Foundation - Flanders (FWO).
* willem.vandenheuvel@chem.kuleuven.be

$\dagger$ liviu.chibotaru@chem.kuleuven.be

1 M. E. Fisher, Phys. Rev. 113, 969 (1959).

2 J. Strečka, Phys. Lett. A 374, 3718 (2010).

3 J. Strečka and M. Jaščur, Czech. J. Phys. 52, A37 (2002).

4 J. Strečka, M. Jaščur, M. Hagiwara, K. Minami, Y. Narumi, and K. Kindo, Phys. Rev. B 72, 024459 (2005).

${ }^{5}$ D. Antonosyan, S. Bellucci, and V. Ohanyan, Phys. Rev. B 79, 014432 (2009).

${ }^{6}$ V. Ohanyan, Condens. Matter Phys. 12, 343 (2009).

7 D. Visinescu, A. M. Madalan, M. Andruh, C. Duhayon, J.-P. Sutter, L. Ungur, W. Van den Heuvel, and L. F. Chibotaru, Chem. Eur. J. 15, 11808 (2009).

8 J. Rinck, G. Novitchi, W. Van den Heuvel, L. Ungur, Y. Lan, C. E. Anson, L. F. Chibotaru, and A. K. Powell, Angew. Chem. Int. Ed. 49, 7583 (2010).

9 For single molecule magnetism see D. Gatteschi, R. Sessoli, and J. Villain, Molecular Nanomagnets (Oxford University Press, Oxford, 2006); for single chain magnetism see C. Coulon, H. Miyasaka, and R. Clérac, in Single-Molecule Magnets and Related Phenomena, Structure and Bonding, Vol. 122, edited by R. Winpenny (Springer-Verlag, Berlin, 2006) pp. 163-206.

10 M. Andruh, J.-P. Costes, C. Diaz, and S. Gao, Inorg. Chem. 48, 3342 (2009).

i1 R. Sessoli and A. K. Powell, Coord. Chem. Rev. 253, 2328 (2009).

12 By complete uniaxial anisotropy we mean that only one $g$-factor of the Kramers doublet is not zero; for example $g_{x}=g_{y}=0$ and $g_{z} \neq 0$.

13 W. P. Wolf, Braz. J. Phys. 30, 794 (2000).
14 In what follows, no use is made of the angular momentum properties of the $s_{i}$. In fact, any parameter that takes on a finite number of values can serve as $s_{i}$ in Eq. (1). In practice however, $s_{i}$ represents most commonly a Kramers doublet, which is most often described as an effective spin$1 / 2$. This is the case for the examples considered in this paper.

15 J. M. Yeomans, Statistical Mechanics of Phase Transitions (Oxford University Press, Oxford, 1992).

16 R. A. Horn and C. R. Johnson, Matrix analysis (Cambridge University Press, Cambridge, 1993).

17 Linear combination in a general sense: the coefficients of the expansion may be operators working in other spaces.

18 P. W. Anderson, Phys. Rev. 115, 2 (1959).

19 For example, "potential exchange" in 1st order, "kinetic exchange" in 2nd order, etc.

20 A. Abragam and B. Bleaney, Electron Paramagnetic Resonance of Transition Ions (Clarendon Press, Oxford, 1970).

21 N. Ishikawa, M. Sugita, T. Okubo, N. Tanaka, T. Iino, and Y. Kaizu, Inorg. Chem. 42, 2440 (2003).

${ }^{22}$ L. F. Chibotaru, L. Ungur, and A. Soncini, Angew. Chem. Int. Ed. 47, 4126 (2008).

${ }^{23}$ L. Ungur, W. Van den Heuvel, and L. F. Chibotaru, New J. Chem. 33, 1224 (2009).

${ }^{24}$ K. Bernot, J. Luzon, L. Bogani, M. Etienne, C. Sangregorio, M. Shanmugam, A. Caneschi, R. Sessoli, and D. Gatteschi, J. Am. Chem. Soc. 131, 5573 (2009).

25 L. Čanová, J. Strečka, and M. Jaščur, J. Phys.:Condens. Matter 18, 4967 (2006).

20 J.-P. Costes, F. Dahan, A. Dupuis, and J.-P. Laurent, Chem. Eur J. 4, 1616 (1998). 
27 O. Kahn, Molecular Magnetism (VCH Publishers, New York, 1993).

28 This result, which was found in Section II C for a periodic ring or chain, is valid here because we are considering the eigenstates of $\mathrm{Dy}_{4} \mathrm{Cr}_{4}$ in the absence of magnetic field, in which case the ring is effectively cyclic symmetric.

29 J. S. Griffith, The Theory of Transition-Metal Ions (Cambridge University Press, Cambridge, 1964). 